\title{
Stability-robustness Analysis for Linear Systems with State-space Models
}

\author{
by HORNG-GIOU CHEN and KUANG-WEI HAN \\ Institute of Electronics, National Chiao-Tung University, Hsinchu, Taiwan, \\ R.O.C.
}

ABSTRACT: The stability-robustness analysis for linear systems with state-space models is considered. The fundamental problem of linear control systems subject to unstructured perturbations is addressed, and the results are extended to the consideration of linear control systems subject to linear structured perturbations. A modified time-domain Lyapunov-based method of stability-robustness analysis is proposed where iterative interpolations of quadratic Lyapunov functions are considered. By use of the proposed method, less conservative allowable perturbation bounds are obtained, and the resulting Lyapunov matrices possess structural informations closely related to the perturbation-susceptible characteristics of the nominal system state matrix. The robustness behaviour of a vertical takeoff and landing (VTOL) aircraft control system, designed by use of the $L Q R$ state-feedback method, is illustrated.

\section{Notation}

We denote the identity matrix by $\mathbf{I}$ and the all zero matrix by $\mathbf{0}$. Given a vector $x \in \mathbb{R}^{n}$, we take $\|x\|=\left(x^{\prime} x\right)^{1 / 2}$. This induces the matrix norm $\|M\|=\sigma_{\max }(M)$ where $\sigma_{\max }(\cdot)$ denotes the operation of taking the largest singular value. The following matrix operations and matrix relations are denoted :

$[\cdot]^{1 / 2} \quad$ square-root of positive-semidefinite matrix

$[\cdot]_{\mathrm{s}} \quad$ symmetric portion of square matrix

$[\cdot]_{\mathrm{m}} \quad$ matrix formed by replacing each entry of a matrix by its modulus value

$[\cdot]_{p s}$ positive-semidefinite matrix formed by replacing each eigenvalue of a symmetric matrix by its modulus value

$\mathbf{P}>0 \quad$ square symmetric matrix $\mathbf{P}$ being positive-definite

$\mathbf{P} \geqslant 0 \quad$ square symmetric matrix $\mathbf{P}$ being positive-semidefinite

$\mathbf{P}>\mathbf{Q}$ square symmetric matrices $\mathbf{P}$ and $\mathbf{Q}$ that satisfy $\mathbf{P}-\mathbf{Q}>0$

$\mathbf{P} \geqslant \mathbf{Q}$ square symmetric matrices $\mathbf{P}$ and $\mathbf{Q}$ that satisfy $\mathbf{P}-\mathbf{Q} \geqslant 0$

\section{Introduction}

In the analysis and design of robust control systems, the fundamental problems are that the assumed mathematical model for the systems are always inexact, and that the parameters of the systems may deviate away from their nominal values. Thus, it is desirable to be able to determine: (i) to what extent a nominal system remains stable when subject to a certain class of perturbations, and (ii) in what way a nominal controller compensator can be adjusted to rectify the perturbation- 
susceptible characteristics of the control system. This is called the quantitative stability-robustness problem (1-17).

The published literature on the quantitative stability-robustness analysis of linear systems can be categorized into two perspectives: (i) the frequency-domain analysis (1-7) which is based on the transfer-function representation of a system, and (ii) the time-domain analysis $(\mathbf{8}-\mathbf{1 6})$ which is based on a state-space representation of a system. The main approach in frequency-domain analysis is to extend the classical single-input single-output stability margins to multiple-input multiple-output systems by use of the singular-value decomposition method. In particular, singularvalue decomposition of the return-difference transfer matrix of a stable feedback control system has been considered, and the tolerable gain and phase changes of an unstructured perturbation in frequency domain has been determined by Mukhopadhyay and Newsom (5). On the other hand, the time-domain approach is more amenable to the consideration of the struclured perturbations in the form of parameter variations and nonlinearities (17). This paper treats the stabilityrobustness analysis in the time domain.

Starting with Patel et al. (8), considerable effort has been given to the reduction of conservatism in time-domain quantitative measures of robustness (9-16). In these contributions, the structural information of the perturbation is algebraically manipulated with the fundamental stability-robustness conditions which were derived for the case of unstructured perturbations. The fundamental stabilityrobustness conditions are the Lyapunov-based result of Patel and Toda (9) and the rootlocus-based result of Qiu and Davison (14), which are the two main techniques of the time-domain robustness analysis. Presently, the rootlocus-based approach (14-16) is known to produce less conservative measures of robustness where linear perturbations are considered. However, the capability of the Lyapunov-based approach $(9-13)$ in dealing with nonlinear time-varying perturbations should not be overlooked. It has been argued that, the robustness measures indirectly derived from the quadratic Lyapunov functions are usually conservative (15). Nevertheless, the Lyapunov-based methods possess the exclusive feature of accompanying the robustness measure with a quadratic Lyapunov function. Thus, research on the Lyapunov-based stability-robustness analysis is conducted in this paper.

In Section II, we present the formulation of the problems concerning the use of quadratic Lyapunov functions for the stability-robustness analysis of linear statespace models with the associated unstructured and structured perturbations. It is shown that, the unstructured robustness-measure problem is fundamental to the stability-robustness analysis of a structurally perturbed system. Once an unstructured robustness-measure problem is solved, a pair of Lyapunov matrices is obtained, then the structured perturbation bounds can be derived by algebraically manipulating the Lyapunov matrices with the structured perturbations. A distinct feature of our approach is that, iterative interpolations of quadratic Lyapunov functions are considered to produce less conservative unstructured robustness measures. The extension of the structured perturbations is similar to the results developed by Zhou and Khargonekar (12), but less conservative robustness measures are achieved for control systems subject to structured perturbations.

In Section III, we show how to apply the interpolations of quadratic Lyapunov 
functions to achieve less conservative unstructured robustness measures. It is a mathematical fact that, the Lyapunov-based unstructured robustness measure is obtained by examining the perturbation-susceptible structure of the resulting Lyapunov matrices. A correct suggestion is that, the resulting Lyapunov matrices possess meaningful information concerning the structural content of perturbations to which the nominal system matrix is particularly susceptible. Thus, instead of producing the unstructured robustness measure alone, the perturbation-susceptible structure of the resulting Lyapunov matrices is explicitly derived. By use of the proposed method, improvements in robustness measures and the properties of the explicitly derived perturbation-susceptible structure are illustrated in Section IV by two examples.

Section V illustrates the application of the proposed robustness analysis to a vertical takeoff and landing (VTOL) aircraft control system which has been designed by use of the LQR state feedback method in both (13) and (18). It is shown that, in reaction to the perturbation-susceptible structure of the VTOL control system, the LQR staı-feedback design can be modified to produce more robust results.

\section{Problem Formulation and the Main Results}

Consider the following dynamical system with perturbations:

$$
\mathrm{d} x(t) / \mathrm{d} t=\mathbf{A} x(t)+f(x(t), t),
$$

where $\mathbf{A} \in \mathbb{R}^{n \times n}$ is the stable nominal system matrix, and $f(x(t), t)$ is a vector perturbing function with $f(0, t)=0$ for all time $t$. The main problem is to study the stability of the perturbed system described by Eq. (1) for various kinds of perturbations. We begin with a review of some results on robust stability due to Patel and Toda (9) and Zhou and Khargonekar (12).

\section{Result 1. Robustness bounds for unstructured perturbations (9)}

Let the perturbing vector function described in Eq. (1) be unstructured perturbations, i.e. an exact expression of $f(x(t), t)$ cannot be written explicitly, and a measure of perturbing magnitude is given by

$$
\mu_{f}=\operatorname{Max}\{\|f(x, t)\| /\|x\|\},
$$

where the Max operates over all $(x, t) \in \mathbb{R}^{n+1}$ with nonzero $x$. It is shown in (9) that the unstructurally perturbed system described by Eqs (1) and (2) is stable if

$$
\mu_{f}<1 / \sigma_{\max }\left(\mathbf{P}_{l}\right) \equiv \mu_{l}
$$

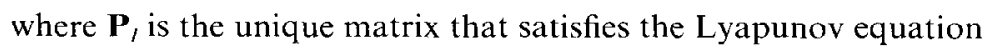

$$
\mathbf{A}^{\prime} \mathbf{P}_{1}+\mathbf{P}_{I} \mathbf{A}=-2 \mathbf{I}
$$

We note that, in the Lyapunov-based analysis of allowable perturbation bounds given in (8-13), the Lyapunov-matrix pair $\left\{\mathbf{P}_{l}, \mathbf{I}\right\}$ in the Lyapunov equation (4) has been adopted for whatever structured perturbations are considered. Thus, the Lyapunov-matrix pair $\left\{\mathbf{P}_{/}, \mathbf{I}\right\}$ constitutes the Lyapunov-based fundamental stability-robustness condition. Followed from the Lyapunov-matrix pair $\left\{\mathbf{P}_{l}, \mathbf{I}\right\}$, 
the Lyapunov-based robustness bounds for structurally perturbed linear dynamical systems are generalized in the work of Zhou and Khargonekar (12).

Result 2. Robustness bounds for linear structured perturbations (12)

Let the perturbing vector function described in Eq. (1) be linear structured perturbations, i.e.

$$
f(x(t), t)=\mathbf{E}(t) x(t)=\sum_{i=1}^{m} k_{i}(t) \mathbf{E}_{i} x(t)
$$

where $\mathbf{E}_{i} \in \mathbb{R}^{n \times n}$ are constant matrices, $k_{i}(t)$ are uncertain time-varying parameters, and the magnitudes of $k_{i}(t)$ are assumed to vary in the intervals around zero, i.e. $k_{i}(t) \in\left[-\varepsilon_{i}, \varepsilon_{i}\right]$. It is shown in (12) that the structurally perturbed system described by Eqs (1) and (5) is stable if,

(i) for $m \geqslant 2$,

$$
\left|k_{j}(t)\right|<1 / \sigma_{\max }\left(\sum_{i=1}^{m}\left[\mathbf{P}_{i}\right]_{m}\right) \equiv \delta_{l}, \quad j=1,2, \ldots, m ;
$$

or

(ii) for $m-1$,

$$
\left|k_{1}(t)\right|<1 / \sigma_{\max }\left(\mathbf{P}_{1}\right),
$$

where matrices $\mathbf{P}_{i}$ are defined by algebraic manipulations of the perturbation matrices $\mathbf{E}_{i}$ given in Eq. (5) with the Lyapunov-matrix pair $\left\{\mathbf{P}_{l}, \mathbf{I}\right\}$ given in the Lyapunov equation (4), i.e.

$$
\mathbf{P}_{i}=\left[\mathbf{P}, \mathbf{E}_{i}\right]_{\mathrm{s}}, \quad i=1,2, \ldots, m .
$$

Instead of confining ourselves to work within the fundamental stability-robustness condition specified by the Lyapunov-matrix pair $\left\{\mathbf{P}_{l}, \mathbf{I}\right\}$ in the Lyapunov equation (4), our main result is started with the advent of a modified fundamental stability-robustness condition specified by the Lyapunov-matrix pair $\{\mathbf{P}, \mathbf{Q}\}$ such that the following Lyapunov equation is fulfilled:

$$
\mathbf{A}^{\prime} \mathbf{P}+\mathbf{P A}=-2 \mathbf{Q},
$$

where matrix $\mathbf{Q} \in \mathbb{R}^{n \times n}$ is symmetric positive-definite, and matrix $\mathbf{P} \in \mathbb{R}^{n \times n}$ is the symmetric positive-definite solution of the Lyapunov equation. Given Lyapunovmatrix pair $\{\mathbf{P}, \mathbf{Q}\}$ that fulfills the Lyapunov equation (9), the unstructured quantitative measure of robustness is given in the following theorem.

\section{Theorem I}

Given Lyapunov matrices $\mathbf{P}$ and $\mathbf{Q}$ that fulfill the Lyapunov equation (9), the unstructurally perturbed system described by Eqs (1) and (2) is stable if,

$$
\mu_{f} \leqslant \operatorname{Min}\left\{x^{\prime} \mathbf{Q} x /\|\mathbf{P} x\|\right\} \equiv \mu_{\mathrm{L}},
$$

where the Min operates over all $x \in \mathbb{R}^{n}$ with $\|x\|=1$. The unstructured robustness bound $\mu_{\mathrm{L}}$ is also given by 


$$
\mu_{\mathbf{L}} \equiv 1 / \operatorname{Max}\left\{\left\|\mathbf{Q}^{-1 / 2} y\right\|\left\|\mathbf{P} \mathbf{Q}^{-1 / 2} y\right\|\right\},
$$

where the Max operates over all $y \in \mathbb{R}^{n}$ with $\|y\|=1$.

Proof: Since $\mathbf{V}(x)=x^{\prime} \mathbf{P} x$ is a Lyapunov function of the stable nominal system matrix $\mathbf{A}$, a sufficient condition for the stability of the perturbed system (1) is

$$
(\mathbf{A} x+f)^{\prime} \mathbf{P} x+x^{\prime} \mathbf{P}(\mathbf{A} x+f) \leqslant 0,
$$

for all $x \in \mathbb{R}^{n}$ with $\|x\|=1$. Following the Lyapunov equation (9), we have

$$
f^{\prime} \mathbf{P} x \leqslant x^{\prime} \mathbf{Q} x
$$

which is sufficiently justified by

$$
\|f\|\|\mathbf{P} x\| \leqslant x^{\prime} \mathbf{Q} x \text {. }
$$

Given the magnitude of perturbation $\mu_{f}$ defined in Eq. (2), we have

$$
\mu_{f} \leqslant x^{\prime} \mathbf{Q} x /\{\|\mathbf{P} x\|\|x\|\},
$$

and the allowable upper bound on the magnitude of perturbation is given by Eq. (10).

By making the replacement of

$$
x=\mathbf{Q}^{-1 / 2} y /\left\|\mathbf{Q}^{-1 / 2} y\right\|,
$$

the relation given in (15) becomes

$$
\mu_{f} \leqslant 1 /\left\{\left\|\mathbf{Q}^{-1 / 2} y\right\|\left\|\mathbf{P Q} \mathbf{Q}^{-1 / 2} y\right\|\right\},
$$

and the allowable upper bound on the magnitude of perturbation is given by Eq. (11).

\section{Remark (1)}

Given matrices $\mathbf{P}$ and $\mathbf{Q}$ that fulfill the Lyapunov equation (9), the unstructured robustness bound $\mu_{\mathrm{L}}$ defined in Eq. (11) can be determined numerically. Thus, the unstructured quantitative stability-robustness problem amounts to the judicious choice of matrix $\mathbf{Q}$ in the Lyapunov equation (9) such that less conservative unstructured robustness bound $\left(\mu_{\mathrm{L}}\right)$ is achieved. This issue is treated in Section III and the Appendix.

On the other hand, given Lyapunov-matrix pair $\{\mathbf{P}, \mathbf{Q}\}$ that fulfills the Lyapunov equation (9), the quantitative measure of robustness for structurally perturbed linear dynamical systems is given in the following theorem.

\section{Theorem II}

Given matrices $\mathbf{P}$ and $\mathbf{Q}$ that fulfill the Lyapunov equation (9), the structurally perturbed system described by (1) and (5) is stable if

$$
\left|k_{j}(t)\right|<1 / \sigma_{\max }\left(\sum_{i=1}^{m}\left[\mathbf{P}_{i}\right]_{\mathrm{ps}}\right) \equiv \delta_{\mathrm{L}}, \quad j=1,2, \ldots, m
$$

where matrices $\mathbf{P}_{i}$ are defined by algebraic manipulations of the perturbation 
matrices $\mathbf{E}_{i}$ given in Eq. (5) with the Lyapunov-matrix pair $\{\mathbf{P}, \mathbf{Q}\}$ given in the Lyapunov equation (9), i.e.

$$
\mathbf{P}_{i}=\mathbf{Q}^{-1 / 2}\left[\mathbf{P E}_{i}\right]_{\mathrm{s}} \mathbf{Q}^{-1 / 2}, \quad i=1,2, \ldots, m .
$$

Proof: We will show that $\mathbf{V}(x, t)=x^{\prime} \mathbf{P} x$ is a Lyapunov function of the structurally perturbed system under the condition given in Eq. (18).

A simple computation shows that

$$
\mathrm{d} \mathbf{V} / \mathrm{d} t=2 x^{\prime} \mathbf{Q}^{1 / 2}\left(\sum_{i=1}^{m} k_{i}(t) \mathbf{P}_{i}-\mathbf{I}\right) \mathbf{Q}^{1 / 2} x .
$$

It is clear that $\mathrm{d} \mathbf{V} / \mathrm{d} t<0$ if

$$
\sigma_{\max }\left(\sum_{i=1}^{m} k_{i}(t) \mathbf{P}_{i}\right)<1
$$

Note that, for all $i=1,2, \ldots, m$ and for all $\varepsilon \in[-1,1]$, we have

$$
\left[\mathbf{P}_{i}\right]_{\mathrm{ps}}-\varepsilon \mathbf{P}_{i} \geqslant 0 \text {. }
$$

Thus,

$$
\sum_{i=1}^{m}\left|k_{i}(t)\right|\left[\mathbf{P}_{i}\right]_{\mathrm{ps}} \geqslant \sum_{i=1}^{m} k_{i}(t) \mathbf{P}_{i}
$$

and

$$
\operatorname{Max}_{j}\left|k_{j}(t)\right| \sigma_{\max }\left(\sum_{i=1}^{m}\left[\mathbf{P}_{i}\right]_{\mathrm{ps}}\right) \geqslant \sigma_{\max }\left(\sum_{i=1}^{m} k_{i}(t) \mathbf{P}_{i}\right) .
$$

Hence, Fq. (18) implies (21).

\section{Derivation of the Robustness Related Perturbation Structure}

Let the perturbation-susceptible structure of the matrices $\mathbf{P}$ and $\mathbf{Q}$ in the Lyapunov equation (9) be taken into consideration in the analysis of stability robustness, the robustness-related perturbation structure of a nominal system matrix $\mathbf{A}$ is defined in the following definition.

\section{Definition (1)}

Consider a stable nominal system matrix $\mathbf{A}$. Let matrices $\mathbf{P}$ and $\mathbf{Q}$ fulfill the Lyapunov equation (9) and bring forth the unstructured robustness bound $\mu_{\mathrm{L}}$ defined in Eq. (11). The robustness-related perturbation structure of the nominal system matrix $\mathbf{A}$ is the unity-rank matrix $\mu_{\mathrm{L}} v w^{\prime}$, where $v$ and $w$ are unit vectors within $\mathbb{R}^{n}$, such that the Lyapunov function $x^{\prime} \mathbf{P} x$ of the nominal system matrix $\mathbf{A}$ fails being a Lyapunov function of the perturbed system matrix $\mathbf{A}+\mu_{\mathbf{L}} v w^{\prime}$.

Given matrices $\mathbf{P}$ and $\mathbf{Q}$ that fulfill the Lyapunov equation (9), the existence of the robustness-related perturbation structure $\mu_{\mathrm{L}} v w^{\prime}$ can be proved by the following theorem. 


\section{Theorem III}

Let $\mu_{\mathrm{L}}$ be the unstructured robustness bound derived by use of Theorem I with matrices $\mathbf{P}$ and $\mathbf{Q}$ that fulfill the Lyapunov equation (9). There exist unit vectors $v$ and $w$ within $\mathbb{R}^{n}$ such that, treating the unity-rank matrix $\mu_{\mathrm{L}} v w^{\prime}$ as a perturbation to the nominal system matrix $\mathbf{A}$, the function $x^{\prime} \mathbf{P} x$ fails being a Lyapunov function of the perturbed system matrix $\mathbf{A}+\mu_{\mathrm{L}} v w^{\prime}$.

Proof: The unstructured robustness bound $\mu_{\mathrm{L}}$ is given in Eq. (11) for a specific $y \in \mathbb{R}^{n}$ with $\|y\|=1$, i.e.

$$
\mu_{\mathbf{L}}=1 /\left\{\left\|\mathbf{Q}^{-1 / 2} y\right\|\left\|\mathbf{P Q} \mathbf{Q}^{-1 / 2} y\right\|\right\},
$$

and $\mathbf{V}(x)=x^{\prime} \mathbf{P} x$ is a Lyapunov function of the stable nominal system matrix $\mathbf{A}$.

Let unit vectors $v$ and $w$ be chosen as:

$$
v=\mathbf{P Q}^{-1 / 2} y /\left\|\mathbf{P Q}^{-1 / 2} y\right\|,
$$

and

$$
w=\mathbf{Q}^{-1 / 2} y /\left\|\mathbf{Q}^{-1 / 2} y\right\|
$$

Employing the quadratic function $\mathbf{V}(x)$ on the perturbed system matrix $\mathbf{A}+\mu_{\mathrm{L}} v w^{\prime}$, we have

$$
\mathrm{d} \mathbf{V}(x) / \mathrm{d} t=2 x^{\prime}\left(\mathbf{A}^{\prime} \mathbf{P}+\mu_{\mathrm{L}} w v^{\prime} \mathbf{P}\right) x
$$

Following the Lyapunov equation (9), we have

$$
\mathrm{d} \mathbf{V}(x) / \mathrm{d} t=2 x^{\prime}\left(-\mathbf{Q}+\mu_{\mathrm{L}} w v^{\prime} \mathbf{P}\right) x .
$$

We will show that, given relations (25), (26) and (27), the choice of $x=w$ nullifies the right-hand part of Eq. (29) which causes $\mathbf{V}(x)$ to fail being a Lyapunov function of the perturbed system matrix.

Following Eqs (25) and (26), we have the following two relations:

$$
w^{\prime} \mathbf{Q} w=\|y\|^{2} /\left\|\mathbf{Q}^{-1 / 2} y\right\|^{2},
$$

and

$$
v^{\prime} \mathbf{P} w=\left\|\mathbf{P} \mathbf{Q}^{-1 / 2} y\right\| /\left\|\mathbf{Q}^{-1 / 2} y\right\|
$$

Thus,

$$
w^{\prime}\left(-\mathbf{Q}+\mu_{\mathrm{L}} w v^{\prime} \mathbf{P}\right) w=-1 /\left\|\mathbf{Q}^{-1 / 2} y\right\|^{2}+\mu_{\mathbf{L}}\left\|\mathbf{P} \mathbf{Q}^{-1 / 2} y\right\| /\left\|\mathbf{Q}^{-1 / 2} y\right\|=0 .
$$

\section{Remark (2)}

It is shown implicitly in Definition (1) and Theorem III that, given matrices $\mathbf{P}$ and $\mathbf{Q}$ that fulfill the Lyapunov equation (9), the unstructured robustness bound $\left(\mu_{\mathrm{L}}\right)$ is derived by examining the perturbation-susceptible structure of the Lyapunov matrices. The suggestion is that the Lyapunov matrices possess meaningful information concerning the structural content of perturbations to which the nominal system matrix $\mathbf{A}$ is particularly susceptible. Presumptively, the less conservative way the unstructured robustness-measure problem is solved, the closer the robustness- 
related perturbation structure is related to the perturbation-susceptible structure of the nominal system matrix $\mathbf{A}$. This relation is illustrated by examples given in Section IV.

\section{Remark (3)}

Iterative procedures have been devised such that the proper sequential choice of matrix $\mathbf{Q}$ in the Lyapunov equation (9) is made and a less conservative unstructured robustness bound $\left(\mu_{\mathrm{L}}\right)$ is obtained along the process. The proposed procedure is developed with the discovery of some interpolating properties of Lyapunov equations. Theoretical developments and two algorithms for the generation of the Lyapunov matrices $\mathbf{P}$ and $\mathbf{Q}$ are given in the Appendix.

We note that, following Definition (1) and Theorem III, the robustness-related perturbation structure of the nominal system matrix $\mathbf{A}$ is derived by replacing the perturbation $f(x(t), t)$ denoted in the perturbed system (1) by a linear unity-rank perturbation, i.e.

$$
f(x(t), t)=\mu_{\mathrm{L}} v w^{\prime} x(t),
$$

where $v$ and $w$ are unit vectors within $\mathbb{R}^{n}$.

Let $\mathbf{V}(x)=x^{\prime} \mathbf{P} x$ remain a Lyapunov function for the system (1) perturbed by Eq. (33), then

$$
\left(\mathbf{A}+\mu_{\mathrm{L}} v w^{\prime}\right)^{\prime} \mathbf{P}+\mathbf{P}\left(\mathbf{A}+\mu_{\mathrm{L}} v w^{\prime}\right) \leqslant 0 .
$$

Following the Lyapunov equation (9), relation (34) becomes

$$
w v^{\prime} \mathbf{P}+\mathbf{P} v w^{\prime} \leqslant 2 \mu_{\mathrm{L}}^{-1} \mathbf{Q},
$$

or

$$
\mathbf{Q}^{-1 / 2}\left(w v^{\prime} \mathbf{P}+\mathbf{P} v w^{\prime}\right) \mathbf{Q}^{-1 / 2} \leqslant 2 \mu_{\mathrm{L}}^{-1} \mathbf{I} .
$$

Thus, there are two relations to be satisfied for all $x \in \mathbb{R}^{n}$ with $\|x\|=1$, i.e.

$$
x^{\prime} \mathbf{Q}^{-1 / 2} \mathbf{P} v w^{\prime} \mathbf{Q}^{-1 / 2} x \leqslant \mu_{\mathrm{L}}^{-1},
$$

and

$$
w^{\prime} \mathbf{Q}^{-1 / 2} x x^{\prime} \mathbf{Q}^{-1 / 2} \mathbf{P} v \leqslant \mu_{\mathrm{L}}^{-1},
$$

which constitute the procedure for deriving the robustness-related perturbation structure $\mu_{\mathrm{L}} v w^{\prime}$.

\section{Algorithm I}

Given matrices $\mathbf{P}$ and $\mathbf{Q}$ that fulfill the Lyapunov equation (9), we have:

Step 1. Initially, let $x$ be the first singular vector of the matrix $\mathbf{Q}^{-1 / 2} \mathbf{P}^{2} \mathbf{Q}^{-1 / 2}$.

Step 2. Given $x$, let $w$ and $v$ be correspondingly the left first and right first singular vectors of the matrix $\mathbf{Q}^{-1 / 2} x x^{\prime} \mathbf{Q}^{-1 / 2} \mathbf{P}$. Equivalently, we have

$$
\begin{aligned}
& w=\mathbf{Q}^{-1 / 2} x /\left\|\mathbf{Q}^{-1 / 2} x\right\|, \\
& v=\mathbf{P Q}^{-1 / 2} x /\left\|\mathbf{P Q}^{-1 / 2} x\right\|,
\end{aligned}
$$


and an intermediate value of the unstructured robustness bound $\mu_{\mathrm{L}}$ is given by

$$
\mu_{\mathrm{L}}=\left\{\left\|\mathbf{Q}^{-1 / 2} x\right\|\left\|\mathbf{P Q} \mathbf{Q}^{-1 / 2} x\right\|\right\}^{-1} .
$$

Step 3. Following $v$ and $w$ given in Eqs (39) and (40), let new $x$ be selected as the first singular vector of the matrix $\mathbf{Q}^{-1 / 2}\left[\mathbf{P} v w^{\prime}\right]_{\mathrm{s}} \mathbf{Q}^{-1 / 2}$, and repeat Step 2 until a convergent condition is detected.

Algorithm I converges to the condition of $x \in \mathbb{R}^{n}$ being the first singular vector of the matrix $\left[\mathbf{Q}^{-1 / 2} \mathbf{P}^{2} \mathbf{Q}^{-1 / 2} x x^{\prime} \mathbf{Q}^{-1}\right]_{\mathrm{s}}$. The successful convergence of Algorithm I is assured by examining a matrix relation given in the following theorem.

\section{Theorem IV}

Consider the symmetric positive-definite matrix given by

$$
\mathbf{M}=\mathbf{Q}^{-1 / 2} \mathbf{P}^{2} \mathbf{Q}^{-1 / 2} /\left(x^{\prime} \mathbf{Q}^{-1 / 2} \mathbf{P}^{2} \mathbf{Q}^{-1 / 2} x\right)+\mathbf{Q}^{-1} /\left(x^{\prime} \mathbf{Q}^{-1} x\right),
$$

where vector $x \in \mathbb{R}^{n}$ with $\|x\|=1$ and positive-definite matrices $\mathbf{P}, \mathbf{Q} \in \mathbb{R}^{n \times n}$ are given. If $\sigma_{\max }(\mathbf{M})=2$, then, for all $z \in \mathbb{R}^{n}$ with $\|z\|=1$,

$$
\left\|\mathbf{P Q} \mathbf{Q}^{-1 / 2} x\right\|\left\|\mathbf{Q}^{-1 / 2} x\right\| \geqslant\left\|\mathbf{P} \mathbf{Q}^{-1 / 2} z\right\|\left\|\mathbf{Q}^{-1 / 2} z\right\| \text {. }
$$

Proof: Since $x^{\prime} \mathbf{M} x=2$ and $\sigma_{\max }(\mathbf{M})=2$, we have

$$
2 \geqslant z^{+} \mathbf{M} z=\left\|\mathbf{P} \mathbf{Q}^{-1 / 2} z\right\|^{2} /\left\|\mathbf{P} \mathbf{Q}^{-1 / 2} x\right\|^{2}+\left\|\mathbf{Q}^{-1 / 2} z\right\|^{2} /\left\|\mathbf{Q}^{-1 / 2} x\right\|^{2} .
$$

Thus,

$$
\begin{aligned}
& \left\{\left\|\mathbf{P} \mathbf{Q}^{-1 / 2} z\right\| /\left\|\mathbf{P Q}^{-1 / 2} x\right\|-\left\|\mathbf{Q}^{-1 / 2} z\right\| /\left\|\mathbf{Q}^{-1 / 2} x\right\|\right\}^{2} \\
& \quad \leqslant 2-2\left(\left\|\mathbf{P} \mathbf{Q}^{-1 / 2} z\right\| /\left\|\mathbf{P} \mathbf{Q}^{-1 / 2} x\right\|\right)\left(\left\|\mathbf{Q}^{-1 / 2} z\right\| /\left\|\mathbf{Q}^{-1 / 2} x\right\|\right),
\end{aligned}
$$

which makes

$$
\left(\left\|\mathbf{P Q} \mathbf{Q}^{-1 / 2} z\right\|\left\|Q^{-1 / 2} z\right\|\right) /\left(\left\|\mathbf{P} \mathbf{Q}^{-1 / 2} x\right\|\left\|\mathbf{Q}^{-1 / 2} x\right\|\right) \leqslant 1 .
$$

\section{Properties of the Robustness Related Perturbation Structure}

Employing Algorithm A1 (or A2) of the Appendix, matrices $\mathbf{P}$ and $\mathbf{Q}$ that fulfill the Lyapunov cquation (9) are chosen to produce less conscrvative unstructured robustness bound $\mu_{\mathrm{L}}$ defined in Eq. (11). By use of Algorithm I, the perturbationsusceptible structure of the resulting Lyapunov matrices $\mathbf{P}$ and $\mathbf{Q}$ is explicitly derived, while the unstructured robustness bound $\left(\mu_{\mathrm{L}}\right)$ is achieved. Since the quantitative robustness-bound measure problem for unstructurally perturbed systems is formulated with the least knowledge concerning the structural content of the perturbations, the resulting Lyapunov matrices possess structural properties closely related to the perturbation-susceptible characteristics of the nominal system matrix. This relation is illustrated by the use of Example 1.

On the other hand, given the Lyapunov matrices $\mathbf{P}$ and $\mathbf{Q}$, Theorem II is employed to derive the robust stability bounds $\left(\delta_{\mathrm{L}}\right)$ for structurally perturbed 
systems. As long as the robustness-related perturbation structure $\mu_{\mathrm{L} .} v w^{\prime}$ is closely related to the genuine perturbation-susceptible structure of the nominal system matrix $\mathbf{A}$, the result of producing less conservative unstructured robustness bounds $\left(\mu_{\mathrm{L}}\right)$ attributes to the generation of less conservative structured robustness bounds $\left(\delta_{\mathrm{L}}\right)$. By use of the proposed method, Example 2 is given to demonstrate the improvement in the analysis of quantitative robustness measures for perturbed systems described by Eq. (1).

\section{Example 1}

Consider a perturbed system described by Eq. (1) with the nominal system matrix given by

$$
\mathbf{A}=\left[\begin{array}{rr}
-1 & 10 \\
0 & -1
\end{array}\right]
$$

The perturbation-susceptible structure of matrix A can be obtained by observation, which is approximately given by

$$
\mu_{\mathrm{L} 0} v_{0} w_{0}^{\prime}=0.1\left[\begin{array}{l}
0 \\
1
\end{array}\right]\left[\begin{array}{l}
1 \\
0
\end{array}\right] .
$$

Employing Algorithm Al of the Appendix, the iterative process produces a sequence of Lyapunov-matrix pairs $\left\{\mathbf{P}_{i}, \mathbf{Q}_{i}\right\}$ that fulfill the Lyapunov equation (9). Let each pair of the matrices $\mathbf{P}_{i}$ and $\mathbf{Q}_{i}$ be used in Algorithm $\mathbf{I}$, a sequence of the robustness-related perturbation structure $\left\{\mu_{\mathrm{L} i} v_{i} w_{i}^{\prime}\right\}$ [defined in Definition (1); also referred to as the perturbation-susceptible structure of the Lyapunov matrices $\boldsymbol{P}_{i}$ and $\mathbf{Q}_{i}$ ] is obtained. Additionally, a matchness index $\chi \equiv v_{0}^{\prime} v_{i} w_{i}^{\prime} w_{0}$ is computed to indicate the closeness of the robustness-related perturbation structure $\left\{\mu_{L i} v_{i} w_{i}^{\prime}\right\}$ to the genuine perturbation-susceptible structure given in Eq. (48). The results are summarized in Table I.

Note that, by reducing the conservatism in the unstructured robustness bounds

TABLE I

Unstructured robustness measures and values of matchness index for Example 1

\begin{tabular}{ccc}
\hline Iterations & $\mu_{\mathrm{L} i}$ & $\chi$ \\
\hline Initial $\mathbf{Q}=\mathbf{I}$ & 0.0194 & 0.0981 \\
1st & 0.0347 & 0.6342 \\
2nd & 0.0492 & 0.8733 \\
3rd & 0.0616 & 0.9411 \\
4th & 0.0714 & 0.9659 \\
5th & 0.0779 & 0.9760 \\
6th & 0.0813 & 0.9798 \\
7th & 0.0823 & 0.9807 \\
8th & 0.0824 & 0.9808 \\
\hline
\end{tabular}


$\left(\mu_{\mathrm{L}}\right)$, the robustness-related perturbation structure $\mu_{\mathrm{L}} v w^{\prime}$ consistently approaches the genuine perturbation-susceptible characteristics of the stable nominal matrix (i.e. the matchness index $\chi$ approaches 1). By use of the proposed method, the process converges to the robustness-related perturbation structure given by

$$
\mu_{\mathrm{L}} v w^{\prime}=0.0824\left[\begin{array}{l}
0.1381 \\
0.9904
\end{array}\right]\left[\begin{array}{l}
0.9903 \\
0.1392
\end{array}\right] .
$$

Note also that Algorithm A2 of the Appendix converges to the same result in one iteration.

\section{Example 2}

Consider a perturbed system described by Eq. (1) with the nominal system matrix given by

$$
\mathbf{A}=\left[\begin{array}{rr}
-3 & -2 \\
1 & 0
\end{array}\right]
$$

Employing Algorithm A2 of the Appendix, the iterate process converges to a Lyapunov-matrix pair $\{\mathbf{P}, \mathbf{Q}\}$, where

$$
\mathbf{Q}=\left[\begin{array}{ll}
5.2361 & 2.6180 \\
2.6180 & 2.6180
\end{array}\right]
$$

and

$$
\mathbf{P}=\left[\begin{array}{ll}
2.1817 & 1.3090 \\
1.3090 & 3.0544
\end{array}\right]
$$

(A) Unstructurally perturbed case. Let matrices $\mathbf{P}$ and $\mathbf{Q}$ in Eqs (51) and (52) be used in Algorithm I, the unstructured robustness bound $\left(\mu_{\mathrm{L}}\right)$ is obtained. The results are summarized in Table II. Obviously, the proposed robustness bound $\left(\mu_{\mathrm{L}}\right)$ is less conservative than the bound $\left(\mu_{l}\right)$ given by Result 1 from Ref. (9).

(B) Structurally perturbed case. For systems (1) perturbed by a single unityrank linear perturbation, it is advisable to always conduct the stability-robustness analysis using the rootlocus-based techniques (14-16) which give exact bounds of stability robustness. In this example, by use of the Lyapunov-based techniques, structured robustness measures are derived and compared for those rank-2 linear

\begin{tabular}{cc} 
Unstructured & $\begin{array}{c}\text { TABLE II } \\
\text { robustness } \\
\text { Example } 2\end{array}$ \\
\hline $\begin{array}{c}\text { Result } 1 \\
\mu_{t}\end{array}$ & $\begin{array}{c}\text { Theorem I } \\
\mu_{\mathrm{L}}\end{array}$ \\
\hline 0.3820 & 0.4842
\end{tabular}


TABLE III

Structured robustness bounds for Example 2

\begin{tabular}{|c|c|c|c|c|c|}
\hline \multicolumn{4}{|c|}{$E_{i}(i=1,2)$} & $\begin{array}{c}\text { Result } 2 \\
\delta /\end{array}$ & $\begin{array}{c}\text { Theorem II } \\
\delta_{\mathrm{L}}\end{array}$ \\
\hline$\left[\begin{array}{l}1 \\
0\end{array}\right.$ & $\left.\begin{array}{l}0 \\
0\end{array}\right]$ & and & {$\left[\begin{array}{ll}0 & 0 \\
1 & 0\end{array}\right]$} & 0.4805 & 0.7868 \\
\hline$\left[\begin{array}{l}1 \\
0\end{array}\right.$ & $\left.\begin{array}{l}0 \\
0\end{array}\right]$ & and & {$\left[\begin{array}{ll}0 & 1 \\
1 & 0\end{array}\right]$} & 1.0000 & 1.1790 \\
\hline $\begin{array}{l}1 \\
0\end{array}$ & $\left.\begin{array}{l}0 \\
0\end{array}\right]$ & and & {$\left[\begin{array}{ll}0 & 0 \\
0 & 1\end{array}\right]$} & 0.3820 & 0.4973 \\
\hline$\left[\begin{array}{l}0 \\
1\end{array}\right.$ & $\left.\begin{array}{l}0 \\
0\end{array}\right]$ & and & {$\left[\begin{array}{ll}0 & 1 \\
0 & 0\end{array}\right]$} & 0.5000 & 0.7073 \\
\hline$\left[\begin{array}{l}0 \\
1\end{array}\right.$ & $\left.\begin{array}{l}0 \\
0\end{array}\right]$ & and & {$\left[\begin{array}{ll}0 & 0 \\
0 & 1\end{array}\right]$} & 0.3028 & 0.3574 \\
\hline$\left[\begin{array}{l}0 \\
0\end{array}\right.$ & $\left.\begin{array}{l}1 \\
0\end{array}\right]$ & and & {$\left[\begin{array}{ll}0 & 0 \\
0 & 1\end{array}\right]$} & 0.3246 & 0.4288 \\
\hline
\end{tabular}

perturbations where two entries of the nominal system matrix $\mathbf{A}$ in Eq. (50) are independently subject to variations.

Let matrices $\mathbf{P}$ and $\mathbf{Q}$ in Eqs (51) and (52) be used in Theorem II, the structured robustness bound $\left(\delta_{\mathrm{L}}\right)$ is obtained. The results are summarized in Table III. Obviously, the proposed robustness bounds $\left(\delta_{\mathrm{L}}\right)$ are less conservative than the bounds $\left(\delta_{I}\right)$ given by Result 2 from Ref. (12).

\section{Robustness Behaviour of a Vertical Takeoff and Landing Aircraft}

Consider the control system of a vertical takeoff and landing (VTOL) aircraft given in (13) and (18). The linearized model of the VTOL aircraft in the vertical plane is described by

$$
\mathrm{d} x(t) / \mathrm{d} t=(\mathbf{F}+\Delta \mathbf{F}) x(t)+(\mathbf{G}+\Delta \mathbf{G}) u(t) .
$$

The components of the state vector $x \in \mathbb{R}^{4}$ and the control vector $u \in \mathbb{R}^{2}$ are given by

$x_{1} \quad$ horizontal velocity (knots)

$x_{2}$ vertical velocity (knots)

$x_{3}$ pitch rate $\left(\right.$ deg s$\left.^{-1}\right)$

$x_{4}$ pitch angle (deg)

$u_{1}$ "collective" pitch control

$u_{2}$ "longitudinal cyclic" pitch control. 
Essentially, control is achieved by varying the angle of attack with respect to air of the rotor blades. The collective control $u_{1}$ is mainly used for controlling the motion of the aircraft vertically up and down. The longitudinal cyclic control $u_{2}$ is basically used to control the horizontal velocity of the aircraft. For typical loading and flight conditions of the VTOL aircraft at a speed of 135 knots, the matrices $F$ and $\mathbf{G}$ are given by

$$
\mathbf{F}=\left[\begin{array}{cccc}
-0.0366 & 0.0271 & 0.0188 & -0.4555 \\
0.0482 & -1.0100 & 0.0024 & -4.0208 \\
0.1002 & 0.3681 & -0.7070 & 1.4200 \\
0 & 0 & 1 & 0
\end{array}\right]
$$

and

$$
\mathbf{G}=\left[\begin{array}{rc}
0.4422 & 0.1761 \\
3.5446 & -7.5992 \\
-5.5200 & 4.4900 \\
0 & 0
\end{array}\right]
$$

A standard Riccati equation is employed in both (13) and (18) such that the nominal state-feedback control, that stabilizes the nominal closed-loop system, is given by

$$
u(t)=\mathbf{K} x(t)=-(r \mathbf{R})^{-1} \mathbf{G}^{\prime} \mathbf{S} x(t),
$$

where the matrix $\mathbf{S}$ satisfies the algebraic equation

$$
\mathbf{F}^{\prime} \mathbf{S}+\mathbf{S F}+\mathbf{H}=\mathbf{S G}(r \mathbf{R})^{-1} \mathbf{G}^{\prime} \mathbf{S}
$$

and the scalar variable $r$ serves as the design variable while weighting matrices $\mathbf{H}$ and $\mathbf{R}$ are given.

It is known that, even for optimal control problems where the system parameters are completely known, the choice of the weighting matrices $\mathbf{H}$ and $\mathbf{R}$ in the algebraic Riccati equation (57) is not an easy one. A commonly followed procedure, that relates these weighting matrices to the subjective criterion of the pilot, is to make matrices $\mathbf{H}$ and $\mathbf{R}$ diagonal with the elements of the matrices inversely proportional to the square of the maximum allowable variations of the state variables and control variables respectively. Thus, as proposed by Narendra and Tripathi (18), we choose

$$
\mathbf{H}=\left[\begin{array}{cccc}
1 / 25 & 0 & 0 & 0 \\
0 & 1 / 4 & 0 & 0 \\
0 & 0 & 0 & 0 \\
0 & 0 & 0 & 0
\end{array}\right]
$$

and

$$
\mathbf{R}=\left[\begin{array}{cc}
1 / 25 & 0 \\
0 & 1 / 9
\end{array}\right]
$$




\section{Horng-Giou Chen and Kuang-Wei Han}

Applying the proposed method of robustness analysis, the robustness behaviour of the VTOL aircraft control system is given in the following.

(A) Unstructurally perturbed case. If the LQR state feedback is employed, then the nominal closed-loop system matrix is given for each value of $r$ as

$$
\mathbf{A}(r)=\mathbf{F}+\mathbf{G K}=\mathbf{F}-\mathbf{G}(r \mathbf{R})^{-\cdot} \mathbf{G}^{\prime} \mathbf{S} .
$$

Employing Algorithm I and Algorithm A2 of the Appendix on the nominal system matrix $\mathbf{A}(r)$, unstructured robustness bounds $\left(\mu_{\mathrm{L}}\right)$ are obtained and the results are summarized as shown in Fig. 1. Obviously, the proposed robustness bounds $\left(\mu_{\mathrm{L}}\right)$ are less conservative than the bounds $\left(\mu_{l}\right)$ given by Result 1 from Ref. (9).

We note that the unstructured robustness bound $\left(\mu_{\mathrm{L}}\right)$ is optimized for controlweighting parameter $r$ at near 1.34. The attainable unstructured robustness bound is 0.294 . Note also that $r=1$ was arbitrarily selected by Narendra and Tripathi (18) without the robustness analysis shown in Fig. 1.

(B) Structurally perturbed case. As the airspeed changes, significant variations take place in the elements $\mathbf{F}_{32}, \mathbf{F}_{34}$ and $\mathbf{G}_{21}$ of the nominal state matrix $\mathbf{F}$ and the input matrix $\mathbf{G}$ given in Eqs (54) and (55), respectively. As given in (18) that, for range of airspeed from 60 to 170 knots, in-phase variations of elements $\mathbf{F}_{32}, \mathbf{F}_{34}$ and $\mathbf{G}_{21}$ are observed such that

$$
\left|\Delta \mathbf{F}_{32}\right|=0.302 ; \quad\left|\Delta \mathbf{F}_{34}\right|=1.300 ; \quad\left|\Delta \mathbf{G}_{21}\right|=2.567 .
$$

The perturbed closed-loop system is given for each value of $r$ as

$$
\mathrm{d} x(t) / \mathrm{d} t=[\mathbf{A}(r)+\mathbf{E}(r)] x(t),
$$

where $\mathbf{A}(r)$ is given in Eq. (60), and

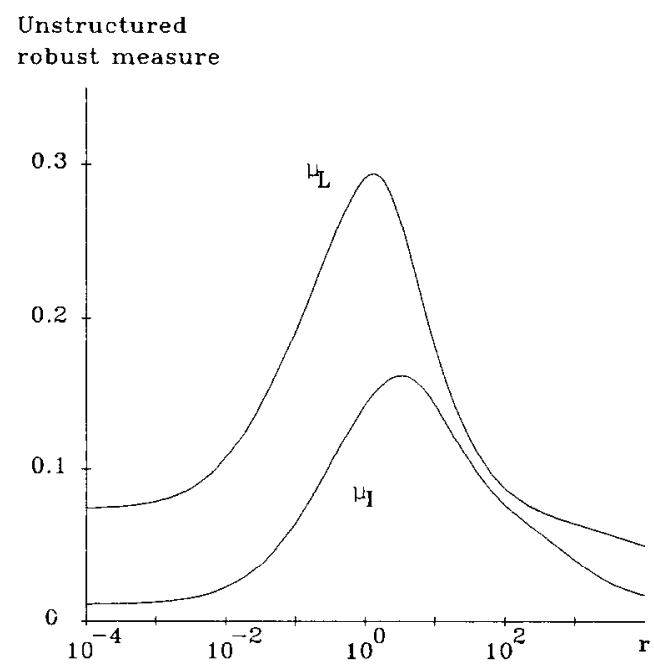

FIG. 1. Unstructured robustness bounds for VTOL aircraft control system designed by use of LQR method with weighting matrices given in Eqs (58) and (59). 


$$
\mathbf{E}(r)=\Delta \mathbf{F}-\Delta \mathbf{G}(r \mathbf{R})^{-1} \mathbf{G}^{\prime} \mathbf{S} .
$$

For in-phase variations given in Eq. (61), the perturbation matrix can be expressed as

$$
\mathbf{E}(r)=k_{\mathrm{i}}(t) \mathbf{E}_{1}=k_{1}(t)\left[\begin{array}{cccc}
0 & 0 & 0 & 0 \\
0 & 0 & 0 & 0 \\
0 & 0.302 & 0 & 1.300 \\
0 & 0 & 0 & 0
\end{array}\right]-k_{1}(t)\left[\begin{array}{cc}
0 & 0 \\
2.567 & 0 \\
0 & 0 \\
0 & 0
\end{array}\right](r \mathbf{R})^{-1} \mathbf{G}^{\prime} \mathbf{S} .
$$

Thus, the matrix pair $\{\mathbf{A}(r), \mathbf{E}(r)\}$ denoted in Eq. (62) constitutes the problem of robustness analysis for structured perturbations.

Employing Theorem II and Algorithm A2 of the Appendix on the structurally perturbed problem specified by the matrix pair $\{\mathbf{A}(r), \mathbf{E}(r)\}$ denoted in Eq. (62), the robustness bounds $\left(\delta_{\mathrm{L}}\right)$ are obtained and the results are summarized in Fig. 2. Obviously, the proposed robustness bounds $\left(\delta_{\mathrm{I}}\right)$ are less conservative than the bounds $\left(\delta_{l}\right)$ given by Result 2 from Ref. (12).

We note that the structured robustness bound $\left(\delta_{L}\right)$ is optimized for controlweighting parameter $r$ at near 160 . The attainable structured robustness bound is 0.939 . Note also that in this example the value of structured robustness bound $\left(\delta_{\mathrm{L}}\right)$ greater than 1 is required to assure the stability of the closed-loop control system described by Eq. (62). Thus, for the design of the LQR state feedback, the weighting matrices [given in Eqs (58) and (59)] proposed by Narendra and Tripathi are not adequate (18).

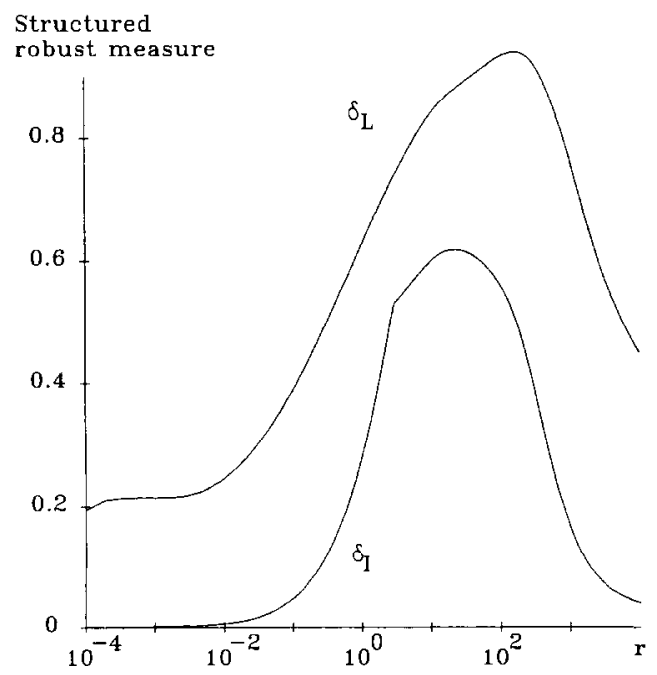

FIG. 2. Structured robustness bounds for VTOL aircraft control system designed by use of LQR method with weighting matrices given in Eqs (58) and (59). 


\section{Horng-Giou Chen and Kuang-Wei Han}

(C) Robustness-related perturbation structure of the VTOL control system. Given weighting matrices specified in Eqs (58) and (59) with weighting parameter $r=1$, the closed-loop nominal system matrix is obtained from $(60)$, i.e.

$$
\mathbf{A}=\left[\begin{array}{cccc}
-0.4421 & -0.3055 & 0.1475 & -0.1275 \\
-0.8779 & -14.2802 & 0.4727 & 0.5195 \\
3.4356 & 12.3970 & -1.8964 & -4.0941 \\
0 & 0 & 1 & 0
\end{array}\right]
$$

Applying Algorithm I and Algorithm A2 of the Appendix, the robustness-related perturbation structure of the matrix $\mathbf{A}$ given in Eq. (65) is derived, i.e.

$$
\mu_{\mathrm{L}} v w^{\prime}=0.2915\left[\begin{array}{r}
0.8715 \\
0.2326 \\
0.2791 \\
-0.3295
\end{array}\right]\left[\begin{array}{r}
0.5552 \\
0.0019 \\
0.8316 \\
-0.0172
\end{array}\right]
$$

which is proposed to represent the perturbation-susceptible structure of the closedloop system matrix A.

Since the stability of the matrix $\mathbf{A}$ given in Eq. (65) is particularly susceptible to the robustness-related perturbation structure given in Eq. (66), then the deficiency of the VTOL aircraft control design can be analysed by examining impulse responses of a system described by :

$$
\mathrm{d} x(t) / \mathrm{d} t=(\mathbf{A}+\mathbf{E}) x(t)+v \delta(t)
$$

and

$$
y(t)=w^{\prime} x(t)
$$

where $\mathbf{A}$ is the matrix given in Eq. (65), $v$ and $w$ are the column matrices that constitute the perturbation-susceptible structure given in $(66), \delta(t)$ is the Dirac impulse function, and $\mathbf{E}$ is the perturbation matrix obtained from (5.12). By use of computer simulation, Figs 3-5 illustrate the impulse responses of the system described by Eqs (67) and (68), i.e. the horizontal velocity $x_{1}$, the vertical velocity $x_{2}$ and the composite output signal $y$. Examining the nominal case $(1.35 \mathrm{Mach})$, it is observed that both $x_{1}$ and $x_{2}$ are well-regulated responses, while $y$ exhibits underdamped characteristics.

Summarizing the examinations in impulse responses, we have :

(i) the LQR state feedback does establish a well-regulated response in accordance with the state weighting matrix $\mathbf{H}$ given in Eq. (58) where $x_{1}$ and $x_{2}$ are proportionally weighted to satisfy the subjective criterion of the pilot;

(ii) the VTOL aircraft control design is deficient in robustness since an underdamped perturbation-susceptible composite output signal $y$ is observed. 


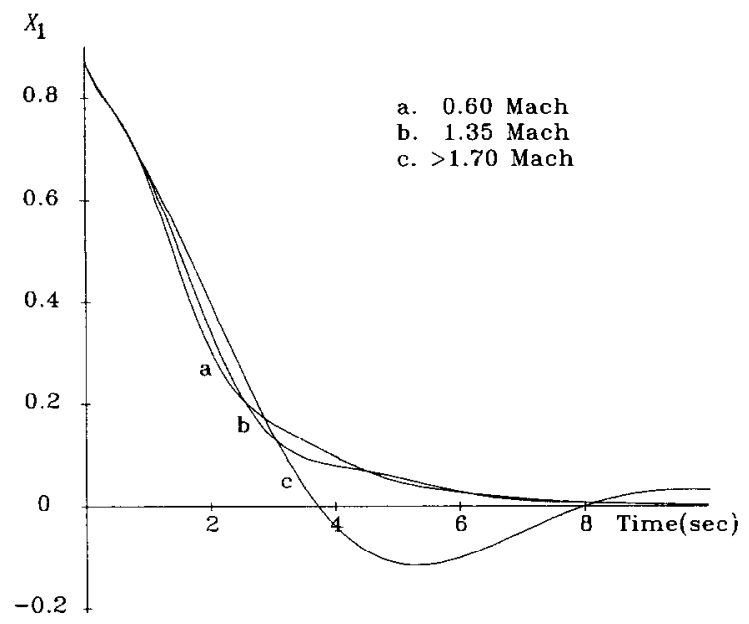

FIG. 3. Impulse response of the horizontal velocity $x_{1}$ for the composite system described by Eqs (67) and (68). The composite system is derived from the control system designed by use of LQR method with weighting matrices given in Eqs (58) and (59).

(D) To robustify the $L Q R$ control design. To robustify the LQR control design of the VTOL aircraft given by Narendra and Tripathi (18), it is suggested that the composite output signal $y$ be weighted in the state weighting matrix instead of $x_{1}$. Compromising the state weightings on $x_{1}$ and on $y$, we consider the state weighting matrix given by

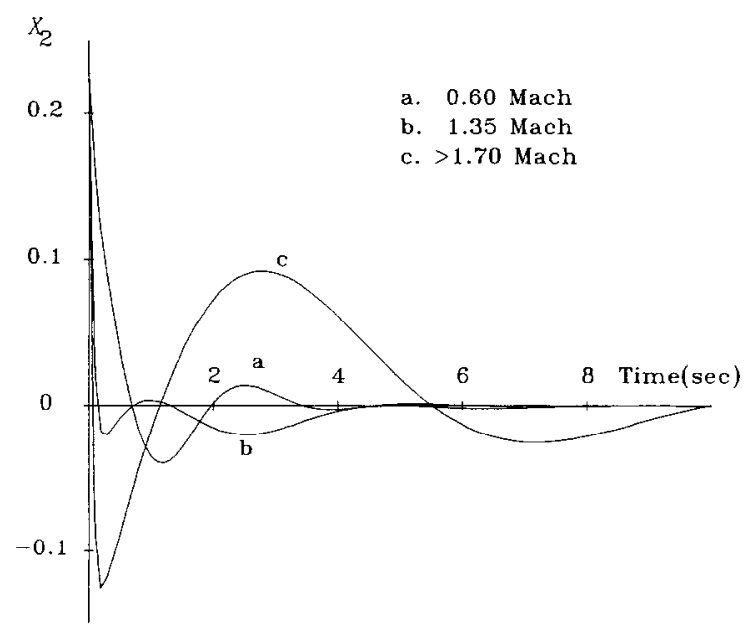

FIG. 4. Impulse response of the vertical velocity $x_{2}$ for the composite system described by Eqs (67) and (68). The composite system is derived from the control system designed by use of LQR method with weighting matrices given in Eqs (58) and (59). 


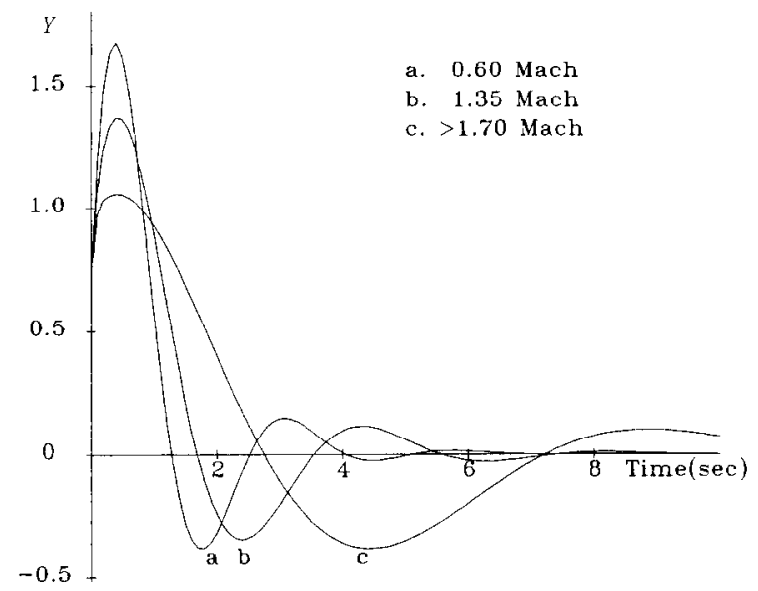

FIG. 5. Impulse response of the composite output signal $y$ for the composite system described by Eqs (67) and (68). The composite system is derived from the control system designed by use of LQR method with weighting matrices given in Eqs (58) and (59).

$$
\mathbf{H}=\left[\begin{array}{cccc}
h_{x 1} & 0 & 0 & 0 \\
0 & 1 / 4 & 0 & 0 \\
0 & 0 & 0 & 0 \\
0 & 0 & 0 & 0
\end{array}\right]+h_{y}\left[\begin{array}{c}
0.5552 \\
0 \\
0.8316 \\
0
\end{array}\right]\left[\begin{array}{c}
0.5552 \\
0 \\
0.8316 \\
0
\end{array}\right]
$$

where

$$
h_{x 1}+h_{y}(0.5552)^{2}--1 / 25 \text {. }
$$

Applying the proposed method of robustness analysis, for various values of the compromising parameter $h_{x \cdot 1}$, the robustness bounds of the modified VTOL aircraft control system are obtained as shown in Figs 6 and 7.

Figure 6 displays the unstructured robustness bounds $\left(\mu_{\mathrm{L}}\right)$, where $h_{x 1}=0.04$ represents the original design. Note that a slight change in the compromising parameter $\left(h_{x 1}\right)$ may give considerable improvement in robustness bounds. Figure 7 displays the structured robustness bounds $\left(\delta_{\mathrm{L}}\right)$. Note that by compromising the state weightings such that $h_{x 1} \rightarrow 0$, the attainable robustness bound is improved.

Thus, selecting $h_{x 1}=0.02$ and $r=0.1$, the modified LQR state-feedback design is derived by assigning a state-weighting matrix as

$$
\mathbf{H}=\left[\begin{array}{cccc}
0.04 & 0 & 0.03 & 0 \\
0 & 0.25 & 0 & 0 \\
0.03 & 0 & 0.0449 & 0 \\
0 & 0 & 0 & 0
\end{array}\right]
$$

The stabilized closed-loop nominal system matrix is given by 


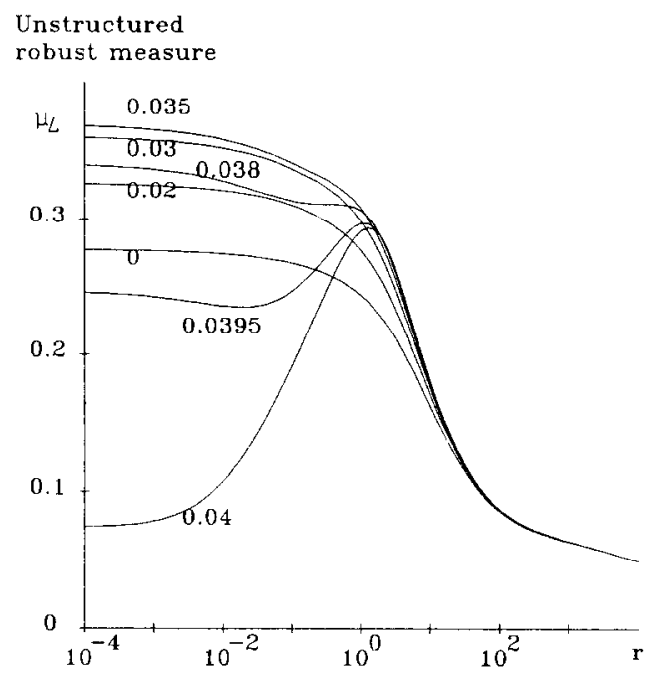

Fig. 6. Unstructured robustness bounds for VTOL aircraft control system designed by use of LQR method with state-weighting matrix given by Eqs (69) and (70) for some values of compromising parameter $h_{x}$.

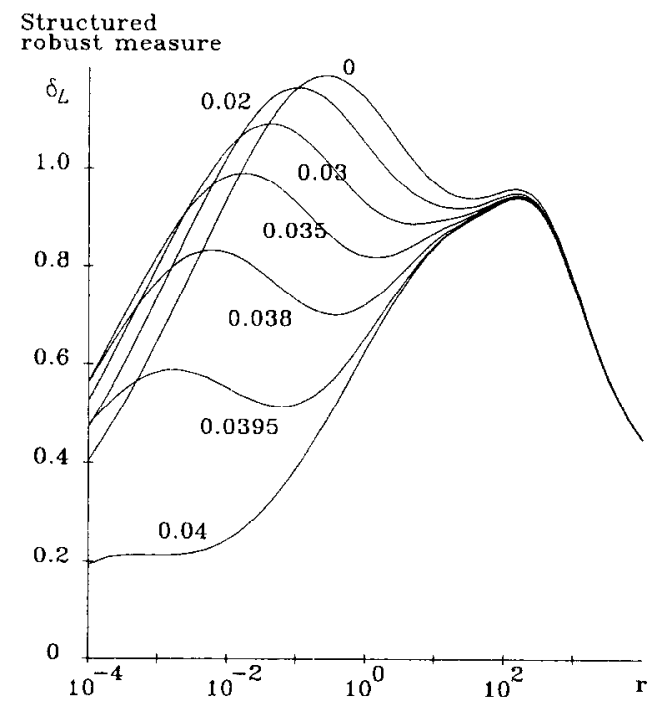

FIG. 7. Structured robustness bounds for VTOI, aircraft control system designed by use of LQR method with state-weighting matrix given by Eqs (69) and (70) for some values of compromising parameter $h_{x 1}$. 


$$
\mathbf{A}=\left[\begin{array}{cccc}
-1.3872 & -0.5166 & 1.2706 & 1.4103 \\
-4.5836 & -43.7041 & 5.6180 & 7.9124 \\
12.3583 & 35.6323 & -13.0510 & -19.6257 \\
0 & 0 & 1 & 0
\end{array}\right]
$$

which possesses the robustness-related perturbation structure given by

$$
\mu_{\mathrm{L}} v w^{\prime}=0.3105\left[\begin{array}{r}
0.9808 \\
0.1107 \\
0.1511 \\
-0.0534
\end{array}\right]\left[\begin{array}{l}
0.8633 \\
0.0217 \\
0.3512 \\
0.3618
\end{array}\right]
$$

and the structured robustness bound is given by

$$
\delta_{\mathrm{L}}=1.1601>1 \text {. }
$$

Since the stability of the matrix A given in (72) is particularly susceptible to the robustness-related perturbation structure given in (73), whether or not the modified LQR control design is better in robustness can be analysed by examining impulse responses of a system described by (67) and (68). Similarly, by use of computer simulation, we have Figs 8-10 to illustrate the impulse responses of $x_{1}, x_{2}$ and $y$. It can be seen that, for all cases, $x_{1}, x_{2}$ and $y$ are well-regulated responses which evidence to justify the robustified LQR control design.

\section{Conclusions}

In this paper, a modified Lyapunov-based method for time-domain stabilityrobustness analysis has been proposed for linear systems with state-space models.

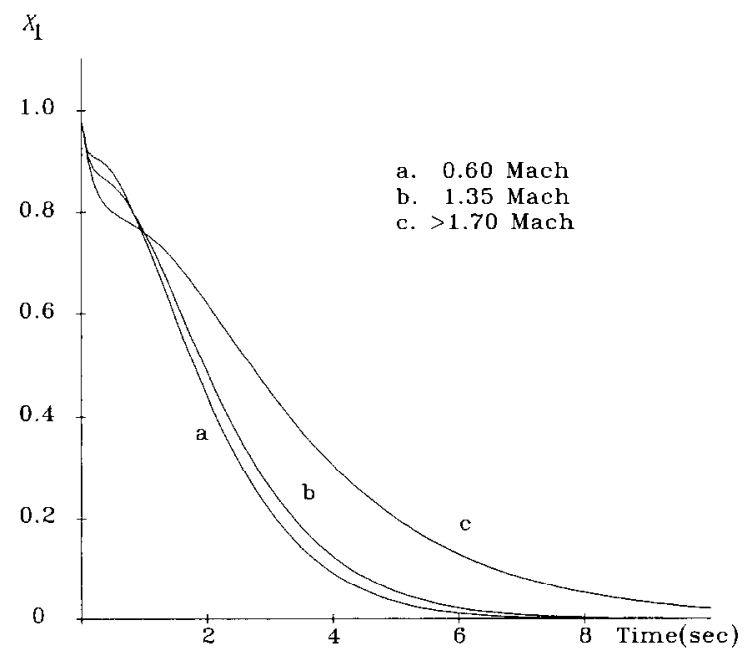

FIG. 8. Impulse response of the horizontal velocity $x_{1}$ for the composite system described by Eqs (67) and (68). The composite system is derived from the control system designed by use of LQR method with state-weighting matrix given by Eq. (71). 


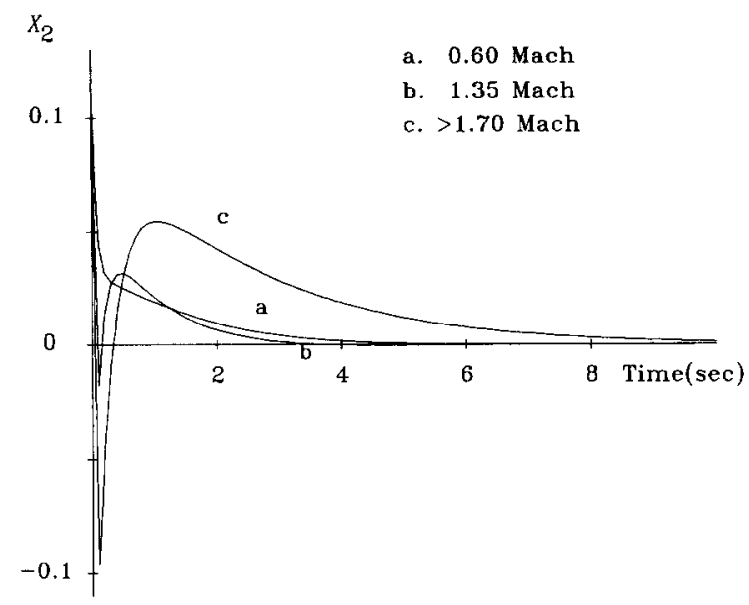

FIG. 9. Impulse response of the vertical velocity $x_{2}$ for the composite system described by Eqs (67) and (68). The composite system is derived from the control system designed by use of LQR method with state-weighting matrix given in Eq. (71).

Following the proposed algorithms, Lyapunov matrices are obtained from which a less conservative quantitative measure of robustness and a scalar internal feedback structure are derived. It has been shown that the derived perturbation-susceptible structure of the Lyapunov matrices is closely related to the perturbation-susceptible

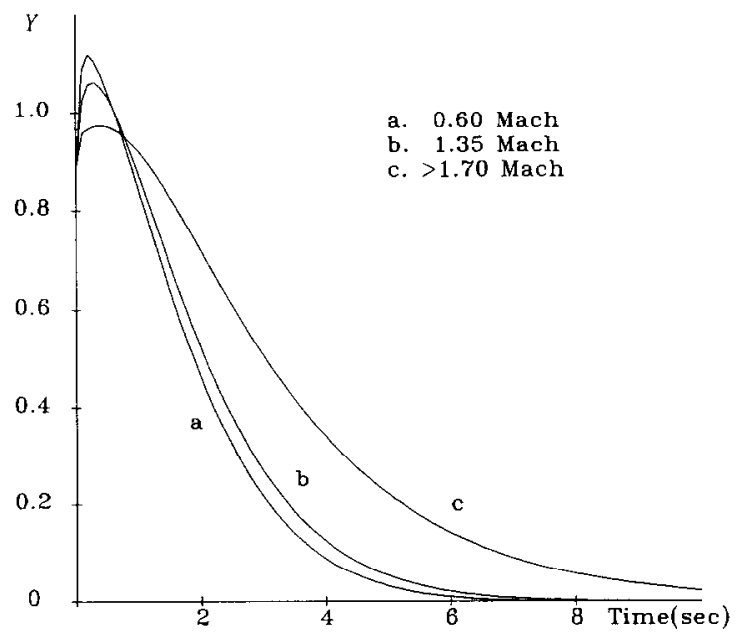

Fig. 10. Impulse response of the composite output signal $y$ for the composite system described by Eqs (67) and (68). The composite system is derived from the control system designed by use of LQR method with state-weighting matrix given in Eq. (71). 
property of the nominal system matrix. In addition, the robustification of a VTOL aircraft state-feedback control system by use of a modified state-weighting matrix has been illustrated.

\section{References}

(1) J. C. Doyle and G. Stein, "Multivariable feedback design: concepts for a classical/ modern synthesis", IEEE Trans. Autom. Control, Vol. AC-26, pp. 4-16, 1981.

(2) I. Postlethwaite, J. M. Edmunds and A. G. J. Macfarlane, "Principal gains and principal phases in the analysis of linear multivariable feedback systems", IEEE Trans. Autom. Control, Vol. AC-26, pp. 32-46, 1981.

(3) M. G. Safonov, A. J. Laub and G. L. Hartmann, "Feedback properties of multivariable systems: the role and use of the return difference matrix", IEEE Trans. Autom. Control, Vol. $\Lambda$ C-26, pp. 47 65, 1981.

(4) N. A. Lehtomaki, N. S. Sandell, Jr and M. Athans. "Robustness results in linear quadratic Gaussian based multivariable control designs", IEEE Trans. Autom. Control, Vol. AC-26, pp. 75 92, 1981.

(5) V. Mukhopadhyay and J. R. Newsom, "A multiloop system stability margin study using matrix singular values", AIAA J. Guidance Control Dynamics, Vol. 7, No. 5, pp. $582587,1984$.

(6) R. W. Daniel and B. Kouvaritakis, "A new robust stability criterion for linear and nonlinear multivariable feedback systems", Int.J. Control, Vol. 41, No. 6, pp. 1349 $1379,1985$.

(7) B. Kouvaritakis and H. Latchman, "Singular value and eigenvalue techniques in the analysis of systems with structured perturbations", Int. J. Control, Vol. 41, No. 6, pp. 1381- 1412, 1985.

(8) R. V. Patel, M. Toda and B. Sridhar, "Robustness of linear quadratic state feedback designs in the presence of system uncertainty", IEEE Trans. Autom. Control, Vol. AC-22, pp. $945949,1977$.

(9) R. V. Patel and M. Toda, "Quantitative measures of robustness for multivariable systems", Proc. Joint Automatic Control Conference, San Francisco, TP8-A, 1980.

(10) R. K. Yedavalli, "Improved measures of stability robustness for linear state space models", IEEE Trans. Autom. Control, Vol. AC-30, pp. 577-579, 1985.

(11) R. K. Yedavalli and Z. Liang, "Reduced conservation in stability robustness bounds by state transformation", IEEE Trans. Autom. Control, Vol. AC-31, pp. 863-866, 1986.

(12) K. Zhou and P. P. Khargonekar, "Stability robustness bounds for linear state space models with structured uncertainty", IEEE Trans. Autom. Control, Vol. AC-32, pp. 621-623, 1987.

(13) R. K. Yedavalli, "Robust control design for aerospace applications", IEEE Trans. Aerospace Electronic Systems, Vol. 25, pp. 314-324, 1989.

(14) L. Qiu and E. J. Davison, "New perturbation bounds for the robust stability of linear state space models", Proc. 25th Conf. on Decision and Control, Athens, Greece, pp. $751-755,1986$.

(15) Y. T. Juang, T. S. Kuo and C. F. Hsu, "New approach to time-domain analysis for stability robustness of dynamic systems", Int. J. Systems Sci., Vol. 18, No. 7, pp. 1363-1376, 1987.

(16) R. K. Yedavalli, "Stability robustness measures under dependent uncertainty", Proc. the American Control Conference, Atlanta, GA, pp. 820-823, 1988. 
(17) D. D. Siljak, "Parameter space methods for robust control design: a guided tour", IEEE Trans. Autom. Control, Vol. AC-34, pp. 674-688, 1989.

(18) K. S. Narendra and S. S. Tripathi, "Identification and optimization of aircraft dynamics", J. Aircraft, Vol. 10, No. 4, pp. 193-199, 1973.

\section{Appendix}

Let $\mathbf{A}$ be a stable system matrix, so $\mathbf{A}^{\prime}$ will be stable. The following Lyapunov equations produce two symmetric positive-definite matrices $\mathbf{P}_{1}$ and $\mathbf{P}_{2}$ :

$$
\begin{aligned}
& \mathbf{A}^{\prime} \mathbf{P}_{1}+\mathbf{P}_{1} \mathbf{A}+2 \mathbf{Q}_{1}=\mathbf{0}, \\
& \mathbf{P}_{2} \mathbf{A}^{\prime}+\mathbf{A} \mathbf{P}_{2}+2 \mathbf{Q}_{2}=\mathbf{0},
\end{aligned}
$$

where matrices $\mathbf{Q}_{1}$ and $\mathbf{Q}_{2}$ are symmetric positive-definite. Simultaneously, we have the following alternative expressions of Eqs (A1) and (A2):

$$
\begin{aligned}
& \mathbf{P}_{1}^{-1} \mathbf{A}^{\prime}+\mathbf{A} \mathbf{P}_{1}^{-1}+2 \mathbf{P}_{1}^{-1} \mathbf{Q}_{1} \mathbf{P}_{1}^{-1}=\mathbf{0}, \\
& \mathbf{A}^{\prime} \mathbf{P}_{2}^{-1}+\mathbf{P}_{2}^{-1} \mathbf{A}+2 \mathbf{P}_{2}^{-1} \mathbf{Q}_{2} \mathbf{P}_{2}^{-1}=\mathbf{0} .
\end{aligned}
$$

Lyapunov equations (A1) and (A4) provide us with two robustness-related perturbation structures. As it is defined in the main text, these perturbation-susceptible structures are $\mu_{1} v_{1} w_{1}^{\prime}$ and $\mu_{2} v_{2} w_{2}^{\prime}$ such that the following relations arc fulfilled:

$$
\begin{aligned}
& \mu_{1}^{-1} v_{1} w_{1}^{\prime}=\mathbf{P}_{1} \mathbf{Q}_{1}^{-1 / 2} x_{1} x_{1}^{\prime} \mathbf{Q}_{1}^{-1 / 2}, \\
& \mu_{2}^{-1} v_{2} w_{2}^{\prime}=\mathbf{Q}_{2}^{-1 / 2} x_{2} x_{2}^{\prime} \mathbf{Q}_{2}^{-1 / 2} \mathbf{P}_{2},
\end{aligned}
$$

where $v_{1}, w_{1}, x_{1}, v_{2}, w_{2}$ and $x_{2}$ are unit vectors within $\mathbb{R}^{n}$, and

$$
\begin{aligned}
& \mu_{1}^{-1}=\left\|\mathbf{P}_{1} \mathbf{Q}_{1}^{-1 / 2} x_{1}\right\|\left\|\mathbf{Q}_{1}^{-1 / 2} x_{1}\right\| \geqslant\left\|\mathbf{P}_{1} \mathbf{Q}_{1}^{-1 / 2} x\right\|\left\|\mathbf{Q}_{1}^{-1 / 2} x\right\|, \\
& \mu_{2}^{-1}=\left\|\mathbf{P}_{2} \mathbf{Q}_{2}^{-1 / 2} x_{2}\right\|\left\|\mathbf{Q}_{2}^{-1 / 2} x_{2}\right\| \geqslant\left\|\mathbf{P}_{2} \mathbf{Q}_{2}^{-1 / 2} x\right\|\left\|\mathbf{Q}_{2}^{-1 / 2} x\right\|,
\end{aligned}
$$

for all $x \in \mathbb{R}^{\prime \prime}$ with $\|x\|=1$.

We note that (A1) and (A4) can be interpolated to make new Lyapunov equations, and that (A2) and (A3) can be interpolated in a dual manner.

\section{Lemma Al}

Given Lyapunov equations (A1) and (A2), the following interpolated Lyapunov equations are satisfied for all interpolating parameters $a, b, c, d>0$, i.e.

$$
\begin{aligned}
& \mathbf{A}^{\prime} \mathbf{X}_{1}+\mathbf{X}_{1} \mathbf{A}+2 \mathbf{Y}_{1}=\mathbf{0}, \\
& \mathbf{X}, \mathbf{A}^{\prime}+\mathbf{A} \mathbf{X}_{2}+2 \mathbf{Y}_{2}=\mathbf{0},
\end{aligned}
$$

where

$$
\begin{aligned}
& \mathbf{X}_{1}=a \mathbf{P}_{1}+b \mathbf{P}_{2}^{-1}, \\
& \mathbf{Y}_{1}=a \mathbf{Q}_{1}+b \mathbf{P}_{2}^{-1} \mathbf{Q}_{2} \mathbf{P}_{2}^{-1}, \\
& \mathbf{X}_{2}=c \mathbf{P}_{2}+d \mathbf{P}_{1}^{-1}, \\
& \mathbf{Y}_{2}=c \mathbf{Q}_{2}+d \mathbf{P}_{1}^{-1} \mathbf{Q}_{1} \mathbf{P}_{1}^{-1} .
\end{aligned}
$$

Proof: Equation (A9) is the direct interpolated result of Eqs (A1) and (A4). Equation (A10) is the direct interpolated result of Eqs (A2) and (A3)

The interpolated Lyapunov equations (A9) and (A10) also provide us with two robust- 


\section{Horng-Giou Chen and Kuang-Wei Han}

ness-related perturbation structures. These perturbation-susceptible structures $v_{1} \bar{v}_{1} \bar{w}_{1}^{\prime}$ and $v_{2} \bar{v}_{2} \bar{w}_{2}^{\prime}$ such that the following relations are fulfilled:

$$
\begin{aligned}
& v_{1}^{-1} \bar{v}_{1} \bar{w}_{1}^{\prime}=\mathbf{X}_{1} \mathbf{Y}_{1}^{-1 / 2} \bar{x}_{1} \bar{x}_{1}^{\prime} \mathbf{Y}_{1}^{-1 / 2}, \\
& v_{2}^{-1} \bar{v}_{2} \bar{w}_{2}^{\prime}=\mathbf{Y}_{2}^{-1 / 2} \bar{x}_{2} \bar{x}_{2}^{\prime} \mathbf{Y}_{2}^{-1 / 2} \mathbf{X}_{2},
\end{aligned}
$$

where $\bar{v}_{1}, \bar{w}_{1}, \bar{x}_{1}, \bar{v}_{2}, \bar{w}_{2}$ and $\bar{x}_{2}$ are unit vectors within $\mathbb{R}^{\prime \prime}$, and

$$
\begin{aligned}
& v_{1}^{-1}=\left\|\mathbf{X}_{1} \mathbf{Y}_{1}^{-1 / 2} \bar{x}_{1}\right\|\left\|\mathbf{Y}_{1}^{-1 / 2} \bar{x}_{1}\right\| \geqslant\left\|\mathbf{X}_{1} \mathbf{Y}_{1}^{-1 / 2} x\right\|\left\|\mathbf{Y}_{1}^{-1 / 2} x\right\|, \\
& v_{2}^{-1}=\left\|\mathbf{X}_{2} \mathbf{Y}_{2}^{-1 / 2} \bar{x}_{2}\right\|\left\|\mathbf{Y}_{2}^{-1 / 2} \bar{x}_{2}\right\| \geqslant\left\|\mathbf{X}_{2} \mathbf{Y}_{2}^{-1 / 2} x\right\|\left\|\mathbf{Y}_{2}^{-1 / 2} x\right\|,
\end{aligned}
$$

for all $x \in \mathbb{R}^{\prime \prime}$ with $\|x\|=1$.

The following Lemmas will be useful when interpolating Lyapunov equations are considered for improving the unstructured robustness measures.

\section{Lemma A2}

Given Lyapunov equations (A1) and (A2) with their robustness-related perturbation structures $\mu_{1} v_{1} w_{1}^{\prime}$ and $\mu_{2} v_{2} w_{2}^{\prime}$, the interpolated Lyapunov equations (A9) and (A10) defined in Lemma A1 produce the robustness-related perturbation structures $v_{1} \bar{v}_{1} \bar{w}_{1}^{\prime}$ and $v_{2} \bar{v}_{2} \bar{w}_{2}^{\prime}$ such that, for all interpolating parameters $a, b, c, d>0$,

$$
\operatorname{Min}\left\{v_{1}, v_{2}\right\} \geqslant \operatorname{Min}\left\{\mu_{1}, \mu_{2}\right\} \text {. }
$$

Proof: We shall prove that $v_{1} \geqslant \operatorname{Min}\left\{\mu_{1}, \mu_{2}\right\}$, and the other relation showing that $v_{2} \geqslant \operatorname{Min}\left\{\mu_{1}, \mu_{2}\right\}$ can be proved in a similar way.

The robustness-related perturbation structure $v_{1} \bar{v}_{1} \bar{w}_{1}^{\prime}$ fulfills the relation given in (A15) Since it is given in (A11) that

$$
\mathbf{X}_{1}=a \mathbf{P}_{1}+b \mathbf{P}_{2}^{-1},
$$

relation (A15) becomes

$$
\begin{aligned}
v_{1}^{-1} \bar{v}_{1} \bar{w}_{1}^{\prime}= & a \mathbf{P}_{1} \mathbf{Y}_{1}^{-1 / 2} \bar{x}_{1} \bar{x}_{1}^{\prime} \mathbf{Y}_{1}^{-1 / 2}+b \mathbf{P}_{2}^{-1} \mathbf{Y}_{1}^{-1 / 2} \bar{x}_{1} \bar{x}_{1}^{\prime} \mathbf{Y}_{1}^{-1 / 2}, \\
= & a \mathbf{P}_{1} \mathbf{Q}_{1}^{-1 / 2} \mathbf{Q}_{1}^{1 / 2} \mathbf{Y}_{1}^{-1 / 2} \bar{x}_{1} \bar{x}_{1}^{\prime} \mathbf{Y}_{1}^{-1 / 2} \mathbf{Q}_{1}^{1 / 2} \mathbf{Q}_{1}^{-1 / 2} \\
& +b \mathbf{Q}_{2}^{-1 / 2} \mathbf{Q}_{2}^{1 / 2} \mathbf{P}_{2}^{-1} \mathbf{Y}_{1}^{-1 / 2} \bar{x}_{1} \bar{x}_{1}^{\prime} \mathbf{Y}_{1}^{-1 / 2} \mathbf{P}_{2}^{-1} \mathbf{Q}_{2}^{1 / 2} \mathbf{Q}_{2}^{-1 / 2} \mathbf{P}_{2} .
\end{aligned}
$$

Defining vectors $y_{1}$ and $z_{1}$ as

$$
\begin{aligned}
& y_{1}=a^{1 / 2} \mathbf{Q}_{1}^{1 / 2} \mathbf{Y}_{1}^{-1 / 2} \bar{x}_{1}, \\
& z_{1}=b^{1 / 2} \mathbf{Q}_{2}^{1 / 2} \mathbf{P}_{2}^{-1} \mathbf{Y}_{1}^{-1 / 2} \bar{x}_{1},
\end{aligned}
$$

relation (A20) becomes

$$
v_{1}^{-1} \bar{v}_{1} \bar{w}_{1}^{\prime}=\mathbf{P}_{1} \mathbf{Q}_{1}^{-1 / 2} y_{1} y_{1}^{\prime} \mathbf{Q}_{1}^{-1 / 2}+\mathbf{Q}_{2}^{-1 / 2} z_{1} z_{1}^{\prime} \mathbf{Q}_{2}^{-1 / 2} \mathbf{P}_{2} .
$$

Thus, by use of the properties given in (A7) and (A8), we have

$$
\begin{aligned}
v_{1}^{-1} & =\sigma_{\max }\left(\mathbf{P}_{1} \mathbf{Q}_{1}^{-1 / 2} y_{1} y_{1}^{\prime} \mathbf{Q}_{1}^{-1 / 2}+\mathbf{Q}_{2}^{-1 / 2} z_{1} z_{1}^{\prime} \mathbf{Q}_{2}^{-1 / 2} \mathbf{P}_{2}\right), \\
& \leqslant\left\|\mathbf{P}_{1} \mathbf{Q}_{1}^{-1 / 2} y_{1}\right\|\left\|\mathbf{Q}_{1}^{-1 / 2} y_{1}\right\|+\left\|\mathbf{Q}_{2}^{-1 / 2} z_{1}\right\|\left\|\mathbf{P}_{2} \mathbf{Q}_{2}^{-1 / 2} z_{1}\right\|, \\
& \leqslant\left\|y_{1}\right\|^{2}\left\|\mathbf{P}_{1} \mathbf{Q}_{1}^{-1 / 2} x_{1}\right\|\left\|\mathbf{Q}_{1}^{-1 / 2} x_{1}\right\|+\left\|z_{1}\right\|^{2}\left\|\mathbf{Q}_{2}^{-1 / 2} x_{2}\right\|\left\|\mathbf{P}_{2} \mathbf{Q}_{2}^{-1 / 2} x_{2}\right\|, \\
& =\left\|y_{1}\right\|^{2} \mu_{1}^{-1}+\left\|z_{1}\right\|^{2} \mu_{2}^{-2} .
\end{aligned}
$$

On the other hand, it is given in (A12) that 


$$
\mathbf{Y}_{1}=a \mathbf{Q}_{1}+b \mathbf{P}_{2}^{-1} \mathbf{Q}_{2} \mathbf{P}_{2}^{-1} .
$$

Followed from (A21) and (A22), it can be shown that

$$
\left\|y_{1}\right\|^{2}+\left\|z_{1}\right\|^{2}=1 \text {. }
$$

Finally, the relation given in (A24) becomes

$$
v_{1}^{-1} \leqslant\left\|y_{1}\right\|^{2} \mu_{1}^{-1}+\left(1-\left\|y_{1}\right\|^{2}\right) \mu_{2}^{-1},
$$

and

$$
v_{1} \geqslant \operatorname{Min}\left\{\mu_{1}, \mu_{2}\right\}
$$

\section{Remark (A1)}

Lemma A2 provides the fact that interpolating Lyapunov equations will not devastate the unstructured robustness bound, i.e. $\operatorname{Min}\left\{v_{1}, v_{2}\right\} \geqslant \operatorname{Min}\left\{\mu_{1}, \mu_{2}\right\}$. The Lyapunov matrices that bring forth a more conservative result are always improved by the Lyapunov matrices that provide a less conservative result.

\section{Lemma A3}

Given Lyapunov equations (A1) and (A2) with their robustness-related perturbation structures $\mu_{1} v_{1} w_{1}^{\prime}$ and $\mu_{2} v_{2} w_{2}^{\prime}$, the interpolated Lyapunov equations (A9) and (A10) defined in Lemma Al produce the robustness-related perturbation structures $v_{1} \bar{v}_{1} \bar{w}_{1}^{\prime}$ and $v_{2} \bar{v}_{2} \bar{w}_{2}^{\prime}$ such that, if either

(1) $\mu_{1} \geqslant \mu_{2}$ and

$$
\mu_{1} v_{1}^{\prime} \mathbf{P}_{2}^{-1} w_{1}<w_{1}^{\prime} \mathbf{P}_{2}^{-1} \mathbf{Q}_{2} \mathbf{P}_{2}^{-1} w_{1},
$$

or (2) $\mu_{2} \geqslant \mu_{1}$ and

$$
\mu_{2} w_{2}^{\prime} \mathbf{P}_{1}^{-1} v_{2}<v_{2}^{\prime} \mathbf{P}_{1}^{-1} \mathbf{Q}_{1} \mathbf{P}_{1}^{-1} v_{2}
$$

then there are some interpolating parameters $a, b, c, d>0$ that make

$$
\operatorname{Max}\left\{v_{1}, v_{2}\right\} \geqslant \operatorname{Max}\left\{\mu_{1}, \mu_{2}\right\} \text {. }
$$

Proof: Lemma A2 implies the result for the case of $\mu_{1}=\mu_{2}$. We shall prove that $\nu_{1} \geqslant \mu_{1}$ while $\mu_{1}>\mu_{2}$, and the remaining relation showing that $\nu_{2} \geqslant \mu_{2}$ while $\mu_{2}>\mu_{1}$ can be proved in a similar way. The proof is divided into two stages:

Stage 1. The interpolated unstructured robustness bound $v_{1}$ fulfills the relation given in (A17), i.e.

$$
v_{1}^{-1}=\operatorname{Max}\left\{\left\|\mathbf{X}_{1} \mathbf{Y}_{1}^{-1 / 2} x\right\|\left\|\mathbf{Y}_{1}^{-1 / 2} x\right\|\right\},
$$

where the Max operates over all $x \in \mathbb{R}^{n}$ with $\|x\|=1$. Since it is given in (A11) that

$$
\mathbf{X}_{1}=a \mathbf{P}_{1}+b \mathbf{P}_{2}^{-1} \text {, }
$$

relation (A17) becomes

$$
v_{1}^{-1}=\operatorname{Max}\left\{\left\|a \mathbf{P}_{1} \mathbf{Y}_{1}^{-1 / 2} x+b \mathbf{P}_{2}^{-1} \mathbf{Y}_{1}^{-1 / 2} x\right\|\left\|\mathbf{Y}_{1}^{-1 / 2} x\right\|\right\} .
$$

Defining the vector $y$ as

$$
y=a^{1 / 2} \mathbf{Q}_{1}^{1 / 2} \mathbf{Y}_{1}{ }^{1 / 2} x,
$$

relation (A31) becomes 
Horng-Giou Chen and Kuang-Wei Han

$$
v_{1}^{-1}=\operatorname{Max}\left\{\left\|\mathbf{P}_{1} \mathbf{Q}_{1}^{-1 / 2} y+(b / a) \mathbf{P}_{2}^{-1} \mathbf{Q}_{1}^{-1 / 2} y\right\|\left\|\mathbf{Q}_{1}^{-1 / 2} y\right\|\right\}
$$

or

$$
v_{1}^{-2}=\operatorname{Max}\left\{y^{\prime} \mathbf{Q}_{1}^{-1 / 2}\left\{\mathbf{P}_{1}+(b / a) \mathbf{P}_{2}^{-1}\right\}^{2} \mathbf{Q}_{1}^{-1 / 2} y y^{\prime} \mathbf{Q}_{1}^{-1} \boldsymbol{y}\right\} .
$$

Thus, the sufficient condition for justifying $\nu_{1} \geqslant \mu_{1}$ is the existence of parameters $a, b>0$ such that

$$
\mu_{1}^{2} y^{\prime} \mathbf{Q}_{1}^{-1 / 2}\left\{\mathbf{P}_{1}+(b / a) \mathbf{P}_{2}^{-1}\right\}^{2} \mathbf{Q}_{1}^{-1 / 2} y y^{\prime} \mathbf{Q}_{1}^{-1} y \leqslant 1,
$$

for all vectors $y \in \mathbb{R}^{\prime \prime}$ defined by (A32).

Since it is given in $(\mathrm{A} / 2)$ that

$$
\mathbf{Y}_{1}=a \mathbf{Q}_{1}+h \mathbf{P}_{2}^{-1} \mathbf{Q}_{2} \mathbf{P}_{2}{ }^{\prime},
$$

then vector $y$ defined in (A32) is restricted by the following relation:

$$
y^{\prime} y+(b / a) y^{\prime} \mathbf{Q}_{1}^{-1 / 2} \mathbf{P}_{2}^{-1} \mathbf{Q}_{2} \mathbf{P}_{2}^{-1} \mathbf{Q}_{1}^{-1 / 2} y=1 .
$$

Thus, the sufficient condition (A35) for justifying $v_{1} \geqslant \mu_{1}$ is equivalently expressed by $\mu_{1}^{2} z^{\prime} \mathbf{Q}_{1}^{-1 / 2}\left\{\mathbf{P}_{1}+(b / a) \mathbf{P}_{2}^{-1}\right\}^{2} \mathbf{Q}_{1}^{-1 / 2} z z^{\prime} \mathbf{Q}_{1}^{-1} z$

$$
\leqslant\left\{z^{\prime} z+(b / a) z^{\prime} \mathbf{Q}_{1}^{-1 / 2} \mathbf{P}_{2}^{-1} \mathbf{Q}_{2} \mathbf{P}_{2}^{-1} \mathbf{Q}_{1}^{-1 / 2} z\right\}^{2},
$$

for all $z \in \mathbb{R}^{n}$ with $\|z\|=1$.

For each vector $z \in \mathbb{R}^{n}$ with $\|z\|=1$, relation (A37) is sufficiently justified by the existence of a scaling factor $s$ such that

$$
\begin{aligned}
s z^{\prime} \mathbf{Q}_{1}^{-1 / 2}\left\{\mathbf{P}_{1}+(b / a) \mathbf{P}_{2}^{-1}\right\}^{2} \mathbf{Q}_{1}^{-1 / 2} z+(1 / s) \mu_{1}^{2} z^{\prime} \mathbf{Q}_{1}^{-1} z & \\
& \leqslant 2 z^{\prime} z+2(b / a) z^{\prime} \mathbf{Q}_{1}^{-1 / 2} \mathbf{P}_{2}^{-1} \mathbf{Q}_{2} \mathbf{P}_{2}^{-1} \mathbf{Q}_{1}^{-1 / 2} z .
\end{aligned}
$$

Collecting terms in (A38) that are multiplied by the powers of $(b / a)$, we have $(b / a)^{2} s z^{\prime} \mathbf{Q}_{1}^{-1 / 2} \mathbf{P}_{2}^{-2} \mathbf{Q}_{1}^{-1,2} z$

$$
\begin{aligned}
+(b / a) z^{\prime} \mathbf{Q}_{1}^{-1 / 2}\left\{s \mathbf{P}_{1} \mathbf{P}_{2}^{-1}+s \mathbf{P}_{2}^{-1} \mathbf{P}_{1}-2 \mathbf{P}_{2}^{-1} \mathbf{Q}_{2} \mathbf{P}_{2}^{-1}\right\} \mathbf{Q}_{1}^{-1 / 2} z \\
\\
\leqslant 2 z^{\prime} z-(1 / s) \mu_{1}^{2} z^{\prime} \mathbf{Q}_{1}^{-1} z-s z^{\prime} \mathbf{Q}_{1}^{-1 / 2} \mathbf{P}_{1}^{2} \mathbf{Q}_{1}^{-1 / 2} z .
\end{aligned}
$$

Denote relation (A39) as

$$
\mathbf{C}_{1}(z)(b / a)^{2}+\mathbf{C}_{2}(z)(b / a) \leqslant \mathbf{C}_{3}(z) .
$$

While $\mathbf{C}_{1}(z)>0$ is recognized, the existence of parameters $a, b>0$ in (A40) is sufficiently justified by either (1) $\mathbf{C}_{3}(z)>0$, or $(2) \mathbf{C}_{2}(z)<0$ with $\mathbf{C}_{3}(z)=0$. In the next stage, we shall prove that coefficients in the polynomial (A40) do fulfill the sufficient condition.

Stage 2. For each vector $z \in \mathbb{R}^{n}$ with $\|z\|=1$, let the scaling factor $s$ be given such that

$$
s z^{\prime} \mathbf{Q}_{1}^{-1 / 2} \mathbf{P}_{1}^{2} \mathbf{Q}_{1}^{-1 / 2} \bar{z}=1 \text {. }
$$

Knowing from (A17) that

$$
\mu_{1}^{-1}=\left\|\mathbf{P}_{1} \mathbf{Q}_{1}^{-1 / 2} x_{1}\right\|\left\|\mathbf{Q}_{1}^{-1 / 2} x_{1}\right\| \geqslant\left\|\mathbf{P}_{1} \mathbf{Q}_{1}^{-1 / 2} z\right\|\left\|\mathbf{Q}_{1}^{-1 / 2} z\right\|,
$$

we have

$$
1 \geqslant \mu_{1}^{2} z^{\prime} \mathbf{Q}_{1}^{-1} z z^{\prime} \mathbf{Q}_{1}^{-1 / 2} \mathbf{P}_{1}^{2} \mathbf{Q}_{1}^{-1 / 2} z-(\mathbf{1} / s) \mu_{1}^{2} z^{\prime} \mathbf{Q}_{1}^{-1} z .
$$

Combining (A41) and (A42), we have 


$$
2 z^{\prime} z-(1 / s) \mu_{1}^{2} z^{\prime} \mathbf{Q}_{1}^{-1} z-s z^{\prime} \mathbf{Q}_{1}^{-1 / 2} \mathbf{P}_{1}^{2} \mathbf{Q}_{1}^{-1 / 2} z \geqslant 0
$$

where the equality holds for the case of $z=x_{1}$.

The condition given in (A28) is

$$
\mu_{1} v_{1}^{\prime} \mathbf{P}_{2}^{-1} w_{1}<w_{1}^{\prime} \mathbf{P}_{2}^{-1} \mathbf{Q}_{2} \mathbf{P}_{2}^{-1} w_{1},
$$

where, by definition given in (A5),

$$
\begin{array}{r}
\mu_{1}^{-1}=\left\|\mathbf{P}_{1} \mathbf{Q}_{1}^{-1 / 2} x_{1}\right\|\left\|\mathbf{Q}_{1}^{-1 / 2} x_{1}\right\|, \\
v_{1}-\mathbf{P}_{1} \mathbf{Q}_{1}^{-1 / 2} x_{1} /\left\|\mathbf{P}_{1} \mathbf{Q}_{1}^{-1 / 2} x_{1}\right\|,
\end{array}
$$

and

$$
w_{1}=\mathbf{Q}_{1}^{-1 / 2} x_{1} /\left\|\mathbf{Q}_{1}^{-1 / 2} x_{1}\right\| .
$$

Thus, relation (A28) becomes

$$
x_{1}^{\prime} \mathbf{Q}_{1}^{-1 / 2} \mathbf{P}_{1} \mathbf{P}_{2}^{-1} \mathbf{Q}_{1}^{-1 / 2} x_{1} /\left\|\mathbf{P}_{1} \mathbf{Q}_{1}^{-1 / 2} x_{1}\right\|^{2}<x_{1}^{\prime} \mathbf{Q}_{1}^{-1 / 2} \mathbf{P}_{2}^{-1} \mathbf{Q}_{2} \mathbf{P}_{2}^{-1} \mathbf{Q}_{1}^{-1 / 2} x_{1}
$$

While $z=x_{1}$, the scaling factor $s$ in (A41) becomes

$$
s x_{1}^{\prime} \mathbf{Q}_{1}^{-1 / 2} \mathbf{P}_{1}^{2} \mathbf{Q}_{1}^{-1 / 2} x_{1}=1,
$$

and relation (A44) leads to

$$
x_{1}^{\prime} \mathbf{Q}_{1}^{-1 / 2}\left\{s \mathbf{P}_{1} \mathbf{P}_{2}^{-1}+s \mathbf{P}_{2}^{-1} \mathbf{P}_{1}-2 \mathbf{P}_{2}^{-1} \mathbf{Q}_{2} \mathbf{P}_{2}^{-1}\right\} \mathbf{Q}_{1}^{-1 / 2} x_{1}<0 .
$$

Therefore, the coefficients of the polynomial (A40) fulfill the sufficient condition for the existence of parameters $a, b>0$ that justify the validity of relation (A39).

\section{Remark (A2)}

Lemma A3 provides the fact that the unstructured robustness bound can be improved by interpolating Lyapunov equations, i.e. $\operatorname{Max}\left\{v_{1}, v_{2}\right\} \geqslant \operatorname{Max}\left\{\mu_{1}, \mu_{2}\right\}$ if a certain numerical property of the Lyapunov matrices is fulfilled. However, the exact values of applicable interpolating parameters remain unknown, and the degree of improvement in the unstructured robustness bound is not predictable. This is a common difficulty of analyses by use of the Lyapunov-based techniques, since the exact numerical property of a Lyapunov equation is not analytically resolvable.

Ncvertheless, the qualitative propcrties of interpolating Lyapunov equations, demonstrated in Lemma A2 and Lemma A3, make it possible to create the following two iterative procedures that produce less conservative unstructured robustness bounds. These algorithms are devised such that, given Lyapunov equations (A1) and (A2) in each itcration, (i) the intermediate values of unstructured robustness bounds $\mu_{1}$ and $\mu_{2}$ are nearly equal to each other, and (ii) Lyapunov equations (A1) and (A2) are interpolated in a balanced manner.

\section{Algorithm Al}

Step 1. Assign $\mathbf{Q}_{1}=\mathbf{Q}_{2}=\mathbf{I}$.

Step 2. Equate Lyapunov equations (A1) and (A2) to acquire the matrices $\mathbf{P}_{1}$ and $\mathbf{P}_{2}$.

Step 3. For $\mathbf{Y}_{1}$ in (A12), interpolating parameters $a$ and $b$ are chosen such that

$$
\sigma_{\min }\left(a \mathbf{Q}_{1}\right)=1
$$

and

$$
\sigma_{\min }\left(b \mathbf{P}_{2}{ }^{1} \mathbf{Q}_{2} \mathbf{P}_{2}^{\prime}\right)=1 .
$$

Similarly, for $\mathbf{Y}_{2}$ in (A14), interpolating parameters $c$ and $d$ are chosen such that 
Horng-Giou Chen and Kuang-Wei Han

$$
\sigma_{\min }\left(c \mathbf{Q}_{2}\right)=1
$$

and

$$
\sigma_{\min }\left(d \mathbf{P}_{1}^{-1} \mathbf{Q}_{1} \mathbf{P}_{1}^{-1}\right)=1
$$

Step 4. Make the replacement of

$$
\mathbf{Q}_{1}=\mathbf{Y}_{1} / \sigma_{\min }\left(\mathbf{Y}_{1}\right)
$$

and

$$
\mathbf{Q}_{2}=\mathbf{Y}_{2} / \sigma_{\min }\left(\mathbf{Y}_{2}\right)
$$

and repeat from Step 2 until a convergent condition is detected.

\section{Algorithm A2}

Step 1. Assign $\mathbf{Q}_{1}=\mathbf{Q}_{2}=\mathbf{I}$.

Step 2. Equate Lyapunov equations (A1) and (A2) to acquire the matrices $\mathbf{P}_{1}$ and $\mathbf{P}_{2}$.

Step 3. For $\mathbf{Y}_{1}$ in (A12), interpolating parameters $a$ and $b$ are chosen such that

$$
\sigma_{\max }\left(a \mathbf{Q}_{1}\right) \sigma_{\min }\left(a \mathbf{Q}_{1}\right)=1 \text {, }
$$

and

$$
\sigma_{\max }\left(b \mathbf{P}_{2}^{-1} \mathbf{Q}_{2} \mathbf{P}_{2}^{-1}\right) \sigma_{\min }\left(b \mathbf{P}_{2}^{-1} \mathbf{Q}, \mathbf{P}_{2}^{-1}\right)=1 .
$$

Similarly, for $\mathbf{Y}_{2}$ in (A14), interpolating parameters $c$ and $d$ are chosen such that

$$
\sigma_{\max }\left(c \mathbf{Q}_{2}\right) \sigma_{\min }\left(C \mathbf{Q}_{2}\right)=1,
$$

and

$$
\sigma_{\max }\left(d \mathbf{P}_{1}^{-1} \mathbf{Q}_{1} \mathbf{P}_{1}^{-1}\right) \sigma_{\min }\left(d \mathbf{P}_{1}^{-1} \mathbf{Q}_{1} \mathbf{P}_{1}^{-1}\right)=1 .
$$

Step 4. Make the replacement of

$$
\mathbf{Q}_{1}=\mathbf{Y}_{1} / \sigma_{\min }\left(\mathbf{Y}_{1}\right)
$$

and

$$
\mathbf{Q}_{2}=\mathbf{Y}_{2} / \sigma_{\min }\left(\mathbf{Y}_{2}\right),
$$

and repeat from Step 2 until a convergent condition is detected.

Received: 30 November 1992

Accepted : 28 February 1993 